\title{
MUSIC-Like Estimation of Direction of Arrival for Noncircular Sources
}

\author{
Habti Abeida and Jean-Pierre Delmas, Senior Member, IEEE
}

\begin{abstract}
This paper examines the asymptotic performance of MUSIC-like algorithms for estimating directions of arrival (DOA) of narrowband complex noncircular sources. Using closed-form expressions of the covariance of the asymptotic distribution of different projection matrices, it provides a unifying framework for investigating the asymptotic performance of arbitrary subspacebased algorithms valid for Gaussian or non-Gaussian and complex circular or noncircular sources. We also derive different robustness properties from the asymptotic covariance of the estimated DOA given by such algorithms. These results are successively applied to four algorithms: to two attractive MUSIC-like algorithms previously introduced in the literature, to an extension of these algorithms, and to an optimally weighted MUSIC algorithm proposed in this paper. Numerical examples illustrate the performance of the studied algorithms compared to the asymptotically minimum variance (AMV) algorithms introduced as benchmarks.
\end{abstract}

Index Terms-Asymptotically minimum variance (AMV), complex noncircular sources, direction of arrival (DOA) estimation, MUSIC algorithm, subspace-based algorithms.

\section{INTRODUCTION}

T HERE is considerable literature about second-order statistics-based algorithms for estimating the direction of arrival (DOA) of narrowband sources impinging on an array of sensors. Among these algorithms, subspace-based algorithms, i.e., algorithms obtained by exploiting the orthogonality between a sample subspace and a DOA parameter-dependent subspace, have been mainly proved very interesting. However, up to now, these algorithms have been designed under the complex circular Gaussian assumption only (see, e.g., [1] and [2]).

In mobile communications, after frequency downshifting the sensor signals to the baseband, the paired in-phase and quadrature components may be complex noncircular [for example, binary-phase-shift-keying (BPSK) and offset-quadrature-phase-shift-keying (OQPSK) modulated signals). Because the second-order statistical characteristics are also contained in the unconjugated spatial covariance matrix for noncircular signals, second-order asymptotically minimum variance (AMV) algorithms [3] and Gaussian maximum-likelihood algorithms [4] must be based on the two covariance matrices. In [3], the potential benefits due to the noncircular property have been evaluated using a closed-form expression of the lower bound on the asymptotic covariance of estimators given by

Manuscript received April 8, 2005; revised July 7, 2005. The associate editor coordinating the review of this manuscript and approving it for publication was Dr. Paul D. Fiore.

The authors are with GET/INT, Département CITI, UMR-CNRS 5157, Institut National des Télécommunications, 91011 Evry Cedex, France (e-mail: jean-pierre.delmas@int-evry.fr,habti.abeida@int-evry.fr).

Digital Object Identifier 10.1109/TSP.2006.873505 arbitrary second-order algorithms. However, the generalized covariance-matching algorithm that attains this bound requires a multidimensional nonlinear optimization, which is computationally demanding. Consequently, we need suboptimal monodimensional optimization algorithms that could benefit from the noncircular property. Such algorithms have been introduced in the context of uncorrelated sources of maximum noncircularity rate impinging on a uniform linear array in [5]-[8], where their performance was observed by simulation only. The aim of this paper is to extend these algorithms, to provide generic asymptotic results for subspace-based estimates of the DOA for noncircular sources based on closed-form expressions of the covariance of the asymptotic distribution of extended projection matrices, and to apply these results to specific MUSIC-like algorithms.

The paper is organized as follows. The array signal model and the statement of the problem are given in Section II. The potential benefit due to the noncircularity property is underscored by the help of subspace-based algorithms built from the unconjugated spatial covariance matrix only in Section III. The four subspace-based algorithms that we shall study are described in Section IV. Their performance is analyzed in Section V using a general functional methodology. Finally, numerical illustrations and Monte Carlo simulations of the performance of the algorithms are given in Section VI.

The following notations are used throughout the paper. Matrices and vectors are represented by bold upper case and bold lower case characters, respectively. Vectors are, by default, in column orientation, while $T, H$, *, and \# stand for transpose, conjugate transpose, conjugate, and Moore Penrose inverse, respectively. $E(\cdot), \operatorname{Tr}(\cdot), \operatorname{Det}(\cdot)\|\cdot\|_{\text {Fro }}, \Re(\cdot)$, and $\Im(\cdot)$ are the expectation, trace, determinant, Frobenius norm, and real and imaginary part operators, respectively. $\mathbf{I}_{K}$ is the identity matrix. $\operatorname{vec}(\cdot)$ is the "vectorization" operator that turns a matrix into a vector by stacking the columns of the matrix one below another, which is used in conjunction with the Kronecker product $\mathbf{A} \otimes \mathbf{B}$ as the block matrix whose $(i, j)$ block element is $a_{i, j} \mathbf{B}$ and with the vec-permutation matrix $\mathbf{K}_{M}$, which transforms $\operatorname{vec}(\mathbf{C})$ to $\operatorname{vec}\left(\mathbf{C}^{T}\right)$ for any $M \times M$ matrix $\mathbf{C}$.

\section{Statement OF THE PROBlem}

Let an array of $M$ sensors receive the signals emitted by $K$ narrowband sources. The observation vectors are modeled as

$$
\mathbf{y}_{t}=\mathbf{A} \mathbf{x}_{t}+\mathbf{n}_{t}, \quad t=1, \ldots, T
$$

where $\left(\mathbf{y}_{t}\right)_{t=1, \ldots, T}$ are independent and identically distributed (i.i.d.). $\mathbf{A}=\left[\mathbf{a}_{1}, \ldots, \mathbf{a}_{K}\right]$ is the steering matrix, where each 
vector $\mathbf{a}_{k}=\mathbf{a}\left(\theta_{k}\right)$ is parameterized by the real scalar parameter $\theta_{k} \cdot \mathbf{x}_{t}=\left(x_{t, 1}, \ldots, x_{t, K}\right)^{T}$ and $\mathbf{n}_{t}$ model signals transmitted by sources and additive measurement noise, respectively. $\mathbf{x}_{t}$ and $\mathbf{n}_{t}$ are multivariate independent, zero-mean; $\mathbf{n}_{t}$ is assumed to be Gaussian complex circular, spatially uncorrelated with $E\left(\mathbf{n}_{t} \mathbf{n}_{t}^{H}\right)=\sigma_{n}^{2} \mathbf{I}_{M}$; while $\mathbf{x}_{t}$ is complex noncircular, not necessarily Gaussian and possibly spatially correlated with nonsingular covariance matrices $\mathbf{R}_{x} \stackrel{\text { def }}{=} E\left(\mathbf{x}_{t} \mathbf{x}_{t}^{H}\right)$ and $\mathbf{R}_{x}^{\prime} \stackrel{\text { def }}{=}$ $E\left(\mathbf{x}_{t} \mathbf{x}_{t}^{T}\right)$. Consequently, this leads to two covariance matrices of $\mathbf{y}_{t}$ that contain information about $\Theta \stackrel{\text { def }}{=}\left(\theta_{1}, \ldots, \theta_{K}\right)^{T}$

$$
\mathbf{R}_{y}=\mathbf{A} \mathbf{R}_{x} \mathbf{A}^{H}+\sigma_{n}^{2} \mathbf{I}_{M} \text { and } \mathbf{R}_{y}^{\prime}=\mathbf{A} \mathbf{R}_{x}^{\prime} \mathbf{A}^{T} \neq \mathbf{O} .
$$

These covariance matrices are traditionally estimated by $\mathbf{R}_{y, T}=(1 / T) \sum_{t=1}^{T} \mathbf{y}_{t} \mathbf{y}_{t}^{H}$ and $\mathbf{R}_{y, T}^{\prime}=(1 / T) \sum_{t=1}^{T} \mathbf{y}_{t} \mathbf{y}_{t}^{T}$, respectively. The parameter vector $\Theta$ is assumed identifiable from $\left(\mathbf{R}_{y}, \mathbf{R}_{y}^{\prime}\right)$.

For a performance analysis, we suppose that the signal waveforms have finite fourth-order moments. The fourth-order cumulants of the sources $\left(x_{t, i}, x_{t, j}, x_{t, k}, x_{t, l}\right)_{i, j, k, l=1, \ldots, K}$ are gathered into the $K^{2} \times K^{2}$ quadrivariance matrix $\mathbf{Q}_{x}$ defined by $\left(\mathbf{Q}_{x}\right)_{i+(j-1) K, k+(l-1) K}=\operatorname{Cum}\left(\left(\mathbf{x}_{t}\right)_{i},\left(\mathbf{x}_{t}\right)_{j}^{*},\left(\mathbf{x}_{t}\right)_{k}^{*},\left(\mathbf{x}_{t}\right)_{l}\right)$. The noncircularity rate $\rho_{k}$ of the $k$ th source is defined by $E\left(x_{t, k}^{2}\right)=\rho_{k} e^{i \phi_{k}} E\left|x_{t, k}^{2}\right|=\rho_{k} e^{i \phi_{k}} \sigma_{k}^{2}$, where $\phi_{k}$ is its noncircularity phase and satisfies $0 \leq \rho_{k} \leq 1$ (from the Cauchy-Schwartz inequality).

The problem addressed in this paper is to estimate the DOA $\Theta$ from the two sample covariance matrices $\mathbf{R}_{y, T}$ and $\mathbf{R}_{y, T}^{\prime}$ by using subspace-based algorithms. The number $K$ of sources is assumed to be known.

\section{Subspace-BASed Algorithms Based on $\mathbf{R}_{y, t}^{\prime}$ Only}

We prove in this section the potential benefit due to the noncircularity property by proposing a MUSIC-like algorithm based on the unconjugated spatial covariance matrix only. Because $\mathbf{R}_{y}^{\prime}$ and $\mathbf{R}_{y}$ have a common noise subspace (see (2.1)) with associated orthogonal projection matrices $\boldsymbol{\Pi}^{\prime}=\boldsymbol{\Pi}$, the first idea for estimating $\Theta$ from $\mathbf{R}_{y, T}^{\prime}$ alone, is to apply the following steps: Estimate the projection matrix $\Pi_{T}^{\prime}$ associated with the noise subspace of $\mathbf{R}_{y, T}^{\prime}$ using the singular value decomposition (SVD) of the symmetric complex-valued matrix $\mathbf{R}_{y, T}^{\prime}$ and, then, use the standard MUSIC algorithm based on $\Pi_{T}^{\prime}$, where the DOA $\left(\theta_{k, T}\right)_{k=1, \ldots, K}$ are estimated as the locations of the $K$ smallest minima of the function

$$
\theta_{k, T}^{\operatorname{Alg}_{0}}=\arg \min _{\theta} g_{0, T}(\theta) \text { with } g_{0, T}(\theta) \stackrel{\text { def }}{=} \mathbf{a}^{H}(\theta) \mathbf{\Pi}_{T}^{\prime} \mathbf{a}(\theta) .
$$

Compared to the standard MUSIC algorithm based on $\Pi_{T}$ associated with the noise subspace of $\mathbf{R}_{y, T}$, whose performance is given e.g., in [1, rel. (3.11a)], we prove in Appendix A the following

Theorem 1: The sequences $\sqrt{T}\left(\Theta_{T}-\Theta\right)$, where $\Theta_{T}$ is the DOA estimate given by these two MUSIC algorithms, converge in distribution to the zero-mean Gaussian distribution of covariance matrix given by

$$
\left(\mathbf{C}_{\Theta}\right)_{k, l}=\frac{2}{\alpha_{k} \alpha_{l}} \Re\left(\left(\mathbf{a}_{l}^{H} \mathbf{U} \mathbf{a}_{k}\right)\left(\mathbf{a}_{k}^{\prime H} \Pi \mathbf{a}_{l}^{\prime}\right)\right)
$$

with $\mathbf{a}_{k}^{\prime} \stackrel{\text { def }}{=}\left(d \mathbf{a}_{k} / d \theta_{k}\right)$ and $\alpha_{k} \stackrel{\text { def }}{=} 2 \mathbf{a}^{\prime}{ }_{k}^{H} \boldsymbol{\Pi} \mathbf{a}_{k}^{\prime}$, where $\mathbf{U} \stackrel{\text { def }}{=}$ $\sigma_{n}^{2} \mathbf{S}^{\#} \mathbf{R}_{y} \mathbf{S}^{\#}$ with $\mathbf{S} \stackrel{\text { def }}{=} \mathbf{A R}_{x} \mathbf{A}^{H}$ and $\mathbf{U} \stackrel{\text { def }}{=} \sigma_{n}^{2} \mathbf{S}^{\prime * \#} \mathbf{R}_{y}^{T} \mathbf{S}^{\prime \#}$ with $\mathbf{S}^{\prime} \stackrel{\text { def }}{=} \mathbf{A} \mathbf{R}_{x}^{\prime} \mathbf{A}^{T}$ for the MUSIC algorithms built on $\mathbf{R}_{y, T}$ and $\mathbf{R}_{y, T}^{\prime}$, respectively.

As a result of the similar structure of $\mathbf{C}_{\Theta}$, given by these two MUSIC algorithms, the asymptotic performance of their estimates can be very similar. In particular, for only one source, it is proved in Appendix A that these asymptotic variances are respectively given by

$$
\begin{aligned}
C_{\theta_{1}} & =\frac{1}{\alpha 1}\left[\frac{\sigma_{n}^{2}}{\sigma_{1}^{2}}+\frac{1}{\left\|\mathbf{a}_{1}\right\|^{2}} \frac{\sigma_{n}^{4}}{\sigma_{1}^{4}}\right] \\
\text { and } C_{\theta_{1}} & =\frac{1}{\alpha_{1} \rho_{1}^{2}}\left[\frac{\sigma_{n}^{2}}{\sigma_{1}^{2}}+\frac{1}{\left\|\mathbf{a}_{1}\right\|^{2}} \frac{\sigma_{n}^{4}}{\sigma_{1}^{4}}\right] .
\end{aligned}
$$

We note that for $\rho_{1}=1$ (e.g., for an unfiltered BPSK modulated source), these two variances are equal. Naturally, when $\rho_{1}$ approaches zero, $C_{\theta_{1}}$ is unbounded and the unconjugated spatial covariance matrix $\mathbf{R}_{y}^{\prime}$ conveys no information about $\theta_{1}$. In consequence of Theorem 1, the following query is raised: How does one combine the statistics $\Pi_{T}$ and $\Pi_{T}^{\prime}$ to improve the estimate of $\Theta$ ? A possible solution is proposed in Section IV.

\section{SubSPACE-BASEd AlgORIthms Under Study}

To devise subspace-based algorithms built from both $\mathbf{R}_{y, T}$ and $\mathbf{R}_{y, T}^{\prime}$, we consider the extended covariance matrix $\mathbf{R}_{\tilde{y}} \stackrel{\text { def }}{=}$ $E\left(\tilde{\mathbf{y}}_{t} \tilde{\mathbf{y}}_{t}^{H}\right)$ where

$$
\tilde{\mathbf{y}}_{t} \stackrel{\text { def }}{=}\left(\begin{array}{c}
\mathbf{y}_{t} \\
\mathbf{y}_{t}{ }^{*}
\end{array}\right)
$$

for which

$$
\mathbf{R}_{\tilde{y}}=\tilde{\mathbf{A}} \mathbf{R}_{\tilde{x}} \tilde{\mathbf{A}}^{H}+\sigma_{n}^{2} \mathbf{I}_{2 M}
$$

with

$$
\tilde{\mathbf{A}} \stackrel{\text { def }}{=}\left(\begin{array}{cc}
\mathbf{A} & \mathbf{O} \\
\mathbf{O} & \mathbf{A}^{*}
\end{array}\right) \text { and } \mathbf{R}_{\tilde{x}} \stackrel{\text { def }}{=}\left(\begin{array}{cc}
\mathbf{R}_{x} & \mathbf{R}_{x}^{\prime} \\
\mathbf{R}_{x}^{* *} & \mathbf{R}_{x}^{*}
\end{array}\right) .
$$

From the assumptions of Section II, $K \leq \operatorname{rank}\left(\mathbf{R}_{\tilde{x}}\right) \leq 2 K$ and depending on this rank, many situations may be considered. We concentrate first on a particular case (case 1) for which the sources are uncorrelated and with noncircularity rate $\rho_{k}$ equal to 1 because very attractive algorithms have been devised for this case [5], [6]. This case corresponds, for example, to unfiltered BPSK or OQPSK uncorrelated modulated signals. In this case, $\mathbf{R}_{x}=\boldsymbol{\Delta}_{\sigma}$ and $\mathbf{R}_{x}^{\prime}=\boldsymbol{\Delta}_{\sigma} \boldsymbol{\Delta}_{\phi}$ with $\boldsymbol{\Delta}_{\sigma} \stackrel{\text { def }}{=} \operatorname{Diag}\left(\sigma_{1}^{2}, \ldots, \sigma_{K}^{2}\right)$ and $\boldsymbol{\Delta}_{\phi} \stackrel{\text { def }}{=} \operatorname{Diag}\left(e^{i \phi_{1}}, \ldots, e^{i \phi_{K}}\right)$. Consequently

$$
\mathbf{R}_{\tilde{x}}=\left(\begin{array}{cc}
\Delta_{\sigma} & \Delta_{\sigma} \Delta_{\phi} \\
\Delta_{\sigma} \Delta_{\phi}^{*} & \Delta_{\sigma}
\end{array}\right)=\left(\begin{array}{c}
\mathbf{I}_{K} \\
\Delta_{\phi}^{*}
\end{array}\right) \boldsymbol{\Delta}_{\sigma}\left(\begin{array}{ll}
\mathbf{I}_{K} & \boldsymbol{\Delta}_{\phi}
\end{array}\right)
$$

and $\operatorname{rank}\left(\mathbf{R}_{\tilde{x}}\right)=K$. Then subsequently, we consider the general case for which $\operatorname{rank}\left(\mathbf{R}_{\tilde{x}}\right)=2 K$ (case 2 ). This case corresponds, for example, to filtered BPSK or OQPSK modulated signals. In these two cases, using the structured matrices $\tilde{\mathbf{A}}$ and $\mathbf{R}_{\tilde{x}}$ (4.2), we prove the following lemma. 
Lemma 1: In cases 1) and 2), the orthogonal projector matrix $\tilde{\Pi}$ onto the noise subspace is structured as

$$
\tilde{\Pi}=\left(\begin{array}{ll}
\Pi_{1} & \Pi_{2} \\
\Pi_{2}^{*} & \Pi_{1}^{*}
\end{array}\right)
$$

where $\Pi_{1}$ and $\Pi_{2}$ are Hermitian and complex symmetric, respectively, and where $\Pi_{1}$ and $\Pi_{2}$ are not projection matrices in case 1 and $\Pi_{1}$ is the orthogonal projector onto the column space of $\mathbf{A}$ and $\Pi_{2}=\mathbf{O}$ in case 2. Furthermore, the orthogonal projector onto the noise subspace $\tilde{\boldsymbol{\Pi}}_{T}$ associated with the sample estimate $\mathbf{R}_{\tilde{y}, T}$ of $\mathbf{R}_{\tilde{y}}$ has the same structure

$$
\tilde{\Pi}_{T}=\left(\begin{array}{ll}
\Pi_{1, T} & \Pi_{2, T} \\
\Pi_{2, T}^{*} & \Pi_{1, T}^{*}
\end{array}\right)
$$

where $\Pi_{1, T}$ and $\Pi_{2, T}$ are Hermitian and complex symmetric respectively.

Proof: Noting that $\mathbf{R}_{\tilde{y}}$ or $\mathbf{R}_{\tilde{y}, T}$ satisfy the relation $\mathbf{R}_{\tilde{y}}=$ $\mathbf{J}_{M} \mathbf{R}_{\tilde{y}}^{*} \mathbf{J}_{M}$ with

$$
\mathbf{J}_{M} \stackrel{\text { def }}{=}\left(\begin{array}{cc}
\mathbf{O}_{M} & \mathbf{I}_{M} \\
\mathbf{I}_{M} & \mathbf{O}_{M}
\end{array}\right)
$$

if $\left(\begin{array}{l}\mathbf{U}_{1} \\ \mathbf{U}_{2}\end{array}\right)$ denotes the partitioned eigenvectors matrix associated with the signal subspace of $\mathbf{R}_{\tilde{y}}$ or $\mathbf{R}_{\tilde{y}, T}$, the corresponding signal part of the eigenvalue decomposition of $\mathbf{R}_{\tilde{y}}$ or $\mathbf{R}_{\tilde{y}, T}$ is

$$
\left(\begin{array}{l}
\mathbf{U}_{1} \\
\mathbf{U}_{2}
\end{array}\right) \boldsymbol{\Sigma}\left(\begin{array}{ll}
\mathbf{U}_{1}^{H} & \mathbf{U}_{2}^{H}
\end{array}\right)=\mathbf{J}_{M}\left(\begin{array}{c}
\mathbf{U}_{1}^{*} \\
\mathbf{U}_{2}^{*}
\end{array}\right) \boldsymbol{\Sigma}\left(\begin{array}{ll}
\mathbf{U}_{1}^{T} & \mathbf{U}_{2}^{T}
\end{array}\right) \mathbf{J}_{M} .
$$

Due to the uniqueness of these normalized eigenvectors up to a unit modulus complex constant, we have $\mathrm{U}_{2}=\mathrm{U}_{1}^{*} \Delta$, where $\Delta$ is a diagonal matrix whose diagonal is composed of unit modulus complex terms. Consequently, these orthogonal projector matrices onto the noise subspace are structured as

$$
\begin{aligned}
\tilde{\mathbf{\Pi}} & =\mathbf{I}_{2 M}-\left(\begin{array}{c}
\mathbf{U}_{1} \\
\mathbf{U}_{2}
\end{array}\right)\left(\begin{array}{ll}
\mathbf{U}_{1}^{H} & \mathbf{U}_{2}^{H}
\end{array}\right) \\
& =\mathbf{I}_{2 M}-\left(\begin{array}{cc}
\mathbf{U}_{1} \mathbf{U}_{1}^{H} & \mathbf{U}_{1} \boldsymbol{\Delta}^{*} \mathbf{U}_{1}^{T} \\
\mathbf{U}_{1}^{*} \boldsymbol{\Delta} \mathbf{U}_{1}^{H} & \mathbf{U}_{1}^{*} \mathbf{U}_{1}^{T}
\end{array}\right) .
\end{aligned}
$$

In case 2 specifically,

$$
\tilde{\mathbf{\Pi}}=\mathbf{I}_{2 M}-\tilde{\mathbf{A}}\left(\tilde{\mathbf{A}}^{H} \tilde{\mathbf{A}}\right)^{-1} \tilde{\mathbf{A}}^{H}=\left(\begin{array}{cc}
\Pi_{1} & \mathbf{O} \\
\mathbf{O} & \Pi_{1}^{*}
\end{array}\right)
$$

with $\Pi_{1} \stackrel{\text { def }}{=} \mathbf{I}_{M}-\mathbf{A}\left(\mathbf{A}^{H} \mathbf{A}\right)^{-1} \mathbf{A}^{H}$.

\section{A. Case 1: Uncorrelated Sources With $\rho_{k}=1$}

Consider now three subspace-based algorithms for case 1 . An algorithm (denoted $\mathrm{Alg}_{1}$ ), devised in [5], has been derived from the standard MUSIC algorithm because in this case (4.1) becomes

$$
\mathbf{R}_{\tilde{y}}=\left(\begin{array}{c}
\mathbf{A} \\
\mathbf{A}^{*} \boldsymbol{\Delta}_{\phi}^{*}
\end{array}\right) \boldsymbol{\Delta}_{\sigma}\left(\begin{array}{ll}
\mathbf{A}^{H} & \left.\boldsymbol{\Delta}_{\phi} \mathbf{A}^{T}\right)^{H}+\sigma_{n}^{2} \mathbf{I}_{2 M} .
\end{array}\right.
$$

Specifically, the estimated DOA $\left(\theta_{k, T}\right)_{k=1, \ldots, K}$ are obtained as the locations of the $K$ smallest minima of the following function:

$$
\theta_{k, T}^{\mathrm{Alg}}=\arg \min _{\theta} g_{1, T}(\theta)
$$

with

$$
\begin{aligned}
g_{1, T}(\theta) & \stackrel{\text { def }}{=} \min _{\phi} \tilde{\mathbf{a}}^{H}(\theta, \phi) \tilde{\Pi}_{T} \tilde{\mathbf{a}}(\theta, \phi) \\
& =\mathbf{a}^{H}(\theta) \boldsymbol{\Pi}_{1, T} \mathbf{a}(\theta)-\left|\mathbf{a}^{T}(\theta) \boldsymbol{\Pi}_{2, T}^{*} \mathbf{a}(\theta)\right|
\end{aligned}
$$

with the extended steering vector

$$
\tilde{\mathbf{a}}(\theta, \phi) \stackrel{\text { def }}{=}\left(\begin{array}{c}
\mathbf{a}(\theta) \\
\mathbf{a}^{*}(\theta) e^{-i \phi}
\end{array}\right) .
$$

Noting that

$$
\tilde{\mathbf{a}}(\theta, \phi)^{H} \tilde{\Pi} \tilde{\mathbf{a}}(\theta, \phi)=\left(\begin{array}{ll}
1 & e^{i \phi}
\end{array}\right) \mathbf{M}\left(\begin{array}{c}
1 \\
e^{-i \phi}
\end{array}\right)=0
$$

with

$$
\mathbf{M} \stackrel{\text { def }}{=}\left(\begin{array}{cc}
\mathbf{a}^{H}(\theta) & \mathbf{0}^{T} \\
\mathbf{0}^{T} & \mathbf{a}^{T}(\theta)
\end{array}\right) \tilde{\mathbf{\Pi}}\left(\begin{array}{cc}
\mathbf{a}(\theta) & \mathbf{0} \\
\mathbf{0} & \mathbf{a}^{*}(\theta)
\end{array}\right),
$$

the matrix

$$
\mathbf{M}_{T} \stackrel{\text { def }}{=}\left(\begin{array}{cc}
\mathbf{a}^{H}(\theta) & \mathbf{0}^{T} \\
\mathbf{0}^{T} & \mathbf{a}^{T}(\theta)
\end{array}\right) \tilde{\mathbf{\Pi}}_{T}\left(\begin{array}{cc}
\mathbf{a}(\theta) & \mathbf{0} \\
\mathbf{0} & \mathbf{a}^{*}(\theta)
\end{array}\right)
$$

is positive definite and a consistent estimate of the rank deficient $2 \times 2$ matrix $\mathbf{M}$. Consequently, we can propose a new subspacebased algorithms (denoted $\mathrm{Alg}_{2}$ ) defined by

$$
\theta_{k, T}^{\mathrm{Alg_{2 }}}=\arg \min _{\theta} g_{2, T}(\theta)
$$

with

$$
\begin{aligned}
g_{2, T}(\theta) \stackrel{\text { def }}{=} & \operatorname{Det}\left(\mathbf{M}_{T}\right) \\
= & \left(\mathbf{a}^{H}(\theta) \boldsymbol{\Pi}_{1, T} \mathbf{a}(\theta)\right)^{2} \\
& -\left(\mathbf{a}^{T}(\theta) \Pi_{2, T}^{*} \mathbf{a}(\theta)\right)\left(\mathbf{a}^{H}(\theta) \boldsymbol{\Pi}_{2, T} \mathbf{a}^{*}(\theta)\right) .
\end{aligned}
$$

In the particular case of a uniform linear array, replacing the generic steering vector $\mathbf{a}(\theta)=\left(1, e^{i \theta}, \ldots, e^{i(M-1) \theta}\right)^{T}$ by $\mathbf{a}(z) \stackrel{\text { def }}{=}\left(1, z, \ldots, z^{M-1}\right)^{T}$ in (4.6), [6] proposed a root-MUSIC-like algorithm (denoted $\mathrm{Alg}_{3}$ ) defined by

$$
\theta_{k, T}^{\operatorname{Alg}_{3}}=\arg \left(z_{k}\right) \text { with } z_{k} K \operatorname{roots}_{|z|<1} \text { of }
$$

$$
g_{3, T}(z) \text { closest to the unit circle }
$$

where $g_{3, T}(z)$ is the following polynomial ${ }^{1}$ of degree $4(M-1)$ whose roots appear in reciprocal conjugate pairs $z_{k}$ and $\left(z_{k}^{*}\right)^{-1}$, as follows:

$$
\begin{aligned}
g_{3, T}(z) \stackrel{\text { def }}{=}\left(\mathbf{a}^{T}\left(z^{-1}\right) \boldsymbol{\Pi}_{1, T} \mathbf{a}(z)\right)^{2} \\
\quad-\left(\mathbf{a}^{T}(z) \boldsymbol{\Pi}_{2, T}^{*} \mathbf{a}(z)\right)\left(\mathbf{a}^{T}\left(z^{-1}\right) \boldsymbol{\Pi}_{2, T} \mathbf{a}\left(z^{-1}\right)\right) .
\end{aligned}
$$

${ }^{1}$ We note that this procedure allows one to estimate up to $2(M-1)$ possible DOA, whereas the upper bound is $2 M-1$ [9]. 


\section{B. Case 2: Arbitrary Full-Rank Spatial Extended Covariance Matrix}

Based on

$$
\tilde{\mathbf{\Pi}} \tilde{\mathbf{A}}=\left(\begin{array}{ll}
\Pi_{1} & \Pi_{2} \\
\Pi_{2}^{*} & \Pi_{1}^{*}
\end{array}\right)\left(\begin{array}{cc}
\mathbf{A} & \mathbf{O} \\
\mathbf{O} & \mathbf{A}^{*}
\end{array}\right)=\mathbf{O}
$$

different MUSIC-like algorithms can be proposed. Since $\boldsymbol{\Pi}_{2}=$ $\mathbf{O}$, a natural idea consists in proposing the following algorithm $\left(\text { denoted } \mathrm{Alg}_{4}\right)^{2}$ :

$$
\theta_{k, T}^{\mathrm{Alg}_{4}}=\arg \min _{\theta} \mathbf{a}^{H}(\theta) \boldsymbol{\Pi}_{1, T} \mathbf{a}(\theta)
$$

It is shown in Section $\mathrm{V}$, however, that this algorithm is always outperformed by the standard MUSIC algorithm based on $\mathbf{R}_{y, T}$ only. Using the ideas of the weighted MUSIC algorithm introduced for DOA estimation [2], then applied for frequency estimation [10], [11], we propose the following column weighting ${ }^{3}$ MUSIC (denoted $\mathrm{Alg}_{5}$ ):

$$
\theta_{k, T}^{\mathrm{Alg} 5_{5}}=\arg \min _{\theta} g_{5, T}(\theta)
$$

with $g_{5, T}(\theta) \stackrel{\text { def }}{=} \operatorname{Tr}\left(\mathbf{W} \overline{\mathbf{A}}^{H}(\theta) \tilde{\boldsymbol{\Pi}}_{T} \overline{\mathbf{A}}(\theta)\right)$, where $\mathbf{W}$ is a $2 \times 2$ nonnegative definite weighting matrix whose optimal value will be specified in Theorem 7, and $\overline{\mathbf{A}}(\theta)$ is the steering matrix

$$
\left(\begin{array}{cc}
\mathbf{a}(\theta) & \mathbf{0} \\
\mathbf{0} & \mathbf{a}^{*}(\theta)
\end{array}\right)
$$

To derive the optimal weighting matrix $\mathbf{W}=\left(\begin{array}{ll}w_{1,1} & w_{1,2} \\ w_{1,2}^{*} & w_{2,2}\end{array}\right)$ in Section V, the weighted MUSIC cost function can be written as

$$
\begin{aligned}
g_{5, T}(\theta)= & \left(w_{1,1}+w_{2,2}\right) \\
& \times\left(\mathbf{a}^{H}(\theta) \Pi_{1, T} \mathbf{a}(\theta)+\Re\left(z \mathbf{a}^{T}(\theta) \boldsymbol{\Pi}_{2, T} \mathbf{a}(\theta)\right)\right)
\end{aligned}
$$

with $z \stackrel{\text { def }}{=}\left(2 w_{1,2}^{*} / w_{1,1}+w_{2,2}\right)$. Consequently, the performance of this algorithm depends only on $z$. By choosing $\mathbf{W}$ diagonal, we have $z=0$, and this algorithm reduces to $\mathrm{Alg}_{4}$.

\section{PERformance AnAlysis}

\section{A. Second-Order Algorithms Based on $\mathbf{R}_{y, t}$ Only}

Considering first the influence of the noncircularity on the performance of an arbitrary second-order algorithm based on $\mathbf{R}_{y, T}$ only, we prove the following theorem.

Theorem 2: All DOA consistent estimates given by an arbitrary second-order algorithms based on $\mathbf{R}_{y, T}$ only, that do not explicitly suppose the sources to be spatially uncorrelated, are robust to the distribution and to the noncircularity of the sources; i.e., the asymptotic performances are those of the standard complex circular Gaussian case.

\footnotetext{
${ }^{2}$ We note that unlike $\Pi_{1}$, the positive semidefinite matrix $\Pi_{1, T}$ is not a projection matrix.

${ }^{3}$ Because $\tilde{\Pi}_{T}$ is an orthogonal projector, the cost function $\left\|\tilde{\mathbf{\Pi}}_{T} \overline{\mathbf{A}}(\theta) \mathbf{W}^{1 / 2}\right\|_{\text {Fro }}^{2}$ reduces to $g_{5, T}(\theta)$.
}

Proof: Based on these assumptions, the Jacobian matrix $\mathbf{D}_{\Theta}^{\mathrm{Alg}}$ of the mapping $\left(\mathbf{R}_{y, T} \longmapsto \Theta_{T}=\operatorname{Alg}\left(\mathbf{R}_{y, T}\right)\right)$ that associates the estimate $\Theta_{T}$ to $\mathbf{R}_{y, T}$ satisfies the constraint (see [12])

$$
\mathbf{D}_{\Theta}^{\mathrm{Alg}}\left(\mathbf{A}^{*} \otimes \mathbf{A}\right)=\mathbf{O}
$$

and because the covariance matrix $\mathbf{C}_{r_{y}}$ of the asymptotic distribution of $\operatorname{vec}\left(\mathbf{R}_{y, T}\right)$ is given by [3]

$$
\begin{aligned}
\mathbf{C}_{r_{y}}=\left(\mathbf{A}^{*} \otimes \mathbf{A}\right) & \mathbf{C}_{r_{x}}\left(\mathbf{A}^{T} \otimes \mathbf{A}^{H}\right)+\sigma_{n}^{4} \mathbf{I}_{M^{2}} \\
& +\sigma_{n}^{2} \mathbf{I}_{M} \otimes \mathbf{A} \mathbf{R}_{x} \mathbf{A}^{H}+\mathbf{A}^{*} \mathbf{R}_{x}^{*} \mathbf{A}^{T} \otimes \sigma_{n}^{2} \mathbf{I}_{M}
\end{aligned}
$$

with $\mathbf{C}_{r_{x}}=\mathbf{R}_{x}^{*} \otimes \mathbf{R}_{x}+\mathbf{K}_{K}\left(\mathbf{R}_{x}^{\prime} \otimes \mathbf{R}_{x}^{* *}\right)+\mathbf{Q}_{x}$, where $\mathbf{Q}_{x}$ is the quadrivariance matrix defined in Section II, the first term of $\mathbf{C}_{r_{y}}$ (which contains $\mathbf{R}_{x}^{\prime}$ and $\mathbf{Q}_{x}$ ) disappears in the expression of the covariance

$$
\mathbf{C}_{\Theta}^{\mathrm{Alg}}=\mathbf{D}_{\Theta}^{\mathrm{Alg}} \mathbf{C}_{r_{y}}\left(\mathbf{D}_{\Theta}^{\mathrm{Alg}}\right)^{H}
$$

of the asymptotic distribution of the estimated DOA $\Theta_{T}$ given by the algorithm $\operatorname{Alg}(\cdot)$.

\section{B. Subspace-Based Algorithms Built From $\mathbf{R}_{\tilde{y}, t}$}

To consider the asymptotic performance of an arbitrary subspace-based algorithms built from $\mathbf{R}_{\tilde{y}, T}$, we adopt a functional analysis which consists of recognizing that the whole process of constructing an estimate $\Theta_{T}$ of $\Theta$ is equivalent to defining a functional relation linking this estimate $\Theta_{T}$ to the statistics $\tilde{\boldsymbol{\Pi}}_{T}$ from which it is inferred. This functional dependence is denoted $\Theta_{T}=\operatorname{Alg}\left(\tilde{\mathbf{\Pi}}_{T}\right)$. By assumption, $\Theta=\operatorname{Alg}(\tilde{\mathbf{\Pi}})$, so arbitrary sufficiently "regular" subspace-based algorithms built from $\mathbf{R}_{\tilde{y}, T}$ constitute distinct extensions of the mapping $\tilde{\boldsymbol{\Pi}} \longmapsto \Theta$. For the different algorithms $\operatorname{Alg}(\cdot)$ defined in Section IV, we note that this mapping is differentiable with respect to $\left(\Pi_{1}, \Pi_{2}, \Pi_{2}^{*}\right)$. With this approach, the asymptotic distributions of the estimates given by these algorithms are directly related to the asymptotic distributions of $\tilde{\boldsymbol{\Pi}}_{T}$ or $\left(\boldsymbol{\Pi}_{1, T}, \boldsymbol{\Pi}_{2, T}, \boldsymbol{\Pi}_{2, T}^{*}\right)$ for which we prove the following theorem in Appendix B.

Theorem 3: The sequence of statistics

$$
\sqrt{T} \operatorname{vec}\left(\tilde{\boldsymbol{\Pi}}_{T}-\tilde{\boldsymbol{\Pi}}\right) \text { and } \sqrt{T}\left(\begin{array}{c}
\operatorname{vec}\left(\boldsymbol{\Pi}_{1, T}-\boldsymbol{\Pi}_{1}\right) \\
\operatorname{vec}\left(\boldsymbol{\Pi}_{2, T}-\boldsymbol{\Pi}_{2}\right) \\
\operatorname{vec}\left(\boldsymbol{\Pi}_{2, T}^{*}-\boldsymbol{\Pi}_{2}^{*}\right)
\end{array}\right)
$$

converge in distribution to the zero-mean Gaussian distributions of first covariance matrices

$$
\mathbf{C}_{\tilde{\Pi}}=\left(\mathbf{I}_{4 M^{2}}+\mathbf{K}_{2 M}\left(\mathbf{J}_{M} \otimes \mathbf{J}_{M}\right)\right)\left(\left(\tilde{\mathbf{\Pi}}^{*} \otimes \tilde{\mathbf{U}}\right)+\left(\tilde{\mathbf{U}}^{*} \otimes \tilde{\mathbf{\Pi}}\right)\right)
$$

and

with

$$
\mathbf{C}_{\Pi_{1}, \Pi_{2}, \Pi_{2}^{*}}=\left(\begin{array}{ccc}
\mathbf{C}_{\Pi_{1}} & \mathbf{C}_{\Pi_{2}, \Pi_{1}}^{H} & \mathbf{C}_{\Pi_{2}^{*}, \Pi_{1}}^{H} \\
\mathbf{C}_{\Pi_{2}, \Pi_{1}} & \mathbf{C}_{\Pi_{2}} & \mathbf{C}_{\Pi_{2}^{*}, \Pi_{2}}^{H} \\
\mathbf{C}_{\Pi_{2}^{*}, \Pi_{1}} & \mathbf{C}_{\Pi_{2}^{*}, \Pi_{2}} & \mathbf{C}_{\Pi_{2}^{*}}^{*}
\end{array}\right)
$$

$$
\begin{aligned}
\mathbf{C}_{\Pi_{1}}= & \left(\boldsymbol{\Pi}_{1}^{*} \otimes \mathbf{U}_{1}\right)+\left(\mathbf{U}_{1}^{*} \otimes \boldsymbol{\Pi}_{1}\right) \\
& +\mathbf{K}_{M}\left(\left(\boldsymbol{\Pi}_{2} \otimes \mathbf{U}_{2}^{*}\right)+\left(\mathbf{U}_{2} \otimes \boldsymbol{\Pi}_{2}^{*}\right)\right) \\
\mathbf{C}_{\Pi_{2}}= & \left(\mathbf{I}_{M^{2}}+\mathbf{K}_{M}\right)\left(\left(\boldsymbol{\Pi}_{1} \otimes \mathbf{U}_{1}\right)+\left(\mathbf{U}_{1} \otimes \boldsymbol{\Pi}_{1}\right)\right) \\
\mathbf{C}_{\Pi_{2}, \Pi_{1}}= & \left(\mathbf{I}_{M^{2}}+\mathbf{K}_{M}\right)\left(\left(\boldsymbol{\Pi}_{2} \otimes \mathbf{U}_{1}\right)+\left(\mathbf{U}_{2} \otimes \boldsymbol{\Pi}_{1}\right)\right) \\
\mathbf{C}_{\Pi_{2}^{*}, \Pi_{1}}= & \left(\mathbf{I}_{M^{2}}+\mathbf{K}_{M}\right)\left(\left(\boldsymbol{\Pi}_{1}^{*} \otimes \mathbf{U}_{2}^{*}\right)+\left(\mathbf{U}_{1}^{*} \otimes \boldsymbol{\Pi}_{2}^{*}\right)\right) \\
\mathbf{C}_{\Pi_{2}^{*}, \Pi_{2}}= & \left(\mathbf{I}_{M^{2}}+\mathbf{K}_{M}\right)\left(\left(\boldsymbol{\Pi}_{2}^{*} \otimes \mathbf{U}_{2}^{*}\right)+\left(\mathbf{U}_{2}^{*} \otimes \boldsymbol{\Pi}_{2}^{*}\right)\right)
\end{aligned}
$$


where $\tilde{\mathbf{U}} \stackrel{\text { def }}{=} \sigma_{n}^{2} \tilde{\mathbf{S}}^{\#} \mathbf{R}_{\tilde{y}} \tilde{\mathbf{S}}^{\#}=\left(\begin{array}{cc}\mathbf{U}_{1} & \mathbf{U}_{2} \\ \mathbf{U}_{2}^{*} & \mathbf{U}_{1}^{*}\end{array}\right)$ with $\tilde{\mathbf{S}} \stackrel{\text { def }}{=}$ $\tilde{\mathbf{A}} \mathbf{R}_{\tilde{x}} \tilde{\mathbf{A}}^{H}$.

We note that Theorem 2 does not extend to arbitrary second-order algorithms based on $\mathbf{R}_{\tilde{y}, T}$ because here $\mathbf{D}_{\Theta}^{\operatorname{Alg}}\left(\tilde{\mathbf{A}}^{*} \otimes \tilde{\mathbf{A}}\right) \neq \mathbf{O}$ due to the constraints on $\mathbf{R}_{\tilde{x}}$ (see the proof in [12]). However, since expression (5.1) of $\mathbf{C}_{\tilde{\Pi}}$ does not depend on the fourth-order moments of the sources, we have proved the following

Theorem 4: The asymptotic performance given by an arbitrary subspace-based algorithm built from $\mathbf{R}_{\tilde{y}, T}$ depends on the distribution of the sources through their second-order moments only.

More specifically, regarding the algorithms described in Section IV, we prove the following.

Theorem 5: The sequences $\sqrt{T}\left(\Theta_{T}-\Theta\right)$, where $\Theta_{T}$ are the DOA estimates given by the first three subspace-based algorithms (respectively, algorithms 1 and 2) described in Section IV for a uniform linear array (respectively, arbitrary array), converge in distribution to the same zero-mean Gaussian distribution ${ }^{4}$ with covariance matrix

$$
\left(\mathbf{C}_{\Theta}\right)_{k, l}=\frac{1}{\gamma_{k} \gamma_{l}}\left(\begin{array}{ll}
\alpha_{\phi, \phi}^{(k)} & -\alpha_{\theta, \phi}^{(k)}
\end{array}\right) \mathbf{B}^{(k, l)}\left(\begin{array}{c}
\alpha_{\phi, \phi}^{(l)} \\
-\alpha_{\theta, \phi}^{(l)}
\end{array}\right)
$$

with $\quad\left(\mathbf{B}^{(k, l)}\right)_{i, j} \quad \stackrel{\text { def }}{=} \quad 4 \Re\left(\left(\tilde{\mathbf{a}}_{k}^{T} \tilde{\mathbf{U}}^{*} \tilde{\mathbf{a}}_{l}^{*}\right)\left(\tilde{\mathbf{a}}_{i, k}^{\prime}{ }_{i, k} \tilde{\mathbf{\Pi}} \tilde{\mathbf{a}}_{j, l}^{\prime}\right)\right)$, $i, j=\theta, \phi$, where

$$
\begin{gathered}
\tilde{\mathbf{a}}_{k} \stackrel{\text { def }}{=}\left(\begin{array}{c}
\mathbf{a}_{k} \\
\mathbf{a}_{k}^{*} e^{-i \phi_{k}}
\end{array}\right) \\
\tilde{\mathbf{a}}_{\theta, k}^{\prime} \stackrel{\text { def }}{=}\left(d \tilde{\mathbf{a}}_{k} / d \theta_{k}\right), \tilde{\mathbf{a}}_{\phi, k}^{\prime} \stackrel{\text { def }}{=}\left(d \tilde{\mathbf{a}}_{k} / d \phi_{k}\right) \text { and with } \\
\left(\alpha_{i, j}^{(k)}\right)_{i, j=\theta, \phi}
\end{gathered}
$$

and $\gamma_{k}$ being the purely geometric factors

$$
\alpha_{i, j}^{(k)} \stackrel{\text { def }}{=} \Re\left(\tilde{\mathbf{a}}_{i, k}^{H}{ }_{i, k}^{H} \tilde{\mathbf{a}}_{j, k}^{\prime}\right)
$$

and $\gamma_{k} \stackrel{\text { def }}{=} \alpha_{\theta, \theta}^{(k)} \alpha_{\phi, \phi}^{(k)}-\left(\alpha_{\theta, \phi}^{(k)}\right)^{2}$. In particular

$$
\left(\mathbf{C}_{\Theta}\right)_{k, k}=\frac{2 \alpha_{\phi, \phi}^{(k)}}{\gamma_{k}}\left(\tilde{\mathbf{a}}_{k}^{H} \tilde{\mathbf{U}}_{k}\right), \quad k=1, \ldots, K
$$

which gives in the case of a single source

$$
C_{\theta_{1}}=\frac{1}{\alpha}\left[\frac{\sigma_{n}^{2}}{\sigma_{1}^{2}}+\frac{1}{2\left\|\mathbf{a}_{1}\right\|^{2}} \frac{\sigma_{n}^{4}}{\sigma_{1}^{4}}\right]
$$

where $\alpha_{1}$ is the purely geometric factor $2 \mathbf{a}_{1}^{\prime}{ }^{H} \Pi \mathbf{a}_{1}^{\prime}$ with $\mathbf{a}_{1}^{\prime} \stackrel{\text { def }}{=}$ $\left(d \mathbf{a}_{1} / d \theta_{1}\right)$.

Remark: If the case of a single noncircular complex Gaussian distributed source of maximum noncircularity rate $\left(\rho_{1}=1\right)$, asymptotic variance (5.6) attains the noncircular Gaussian Cramer-Rao bound given in [4]. Consequently, the first three subspace-based algorithms described in Section IV are efficient for a single source.

Proof: First, we note that the cost functions $g_{1, T}(\alpha)$ and $g_{2, T}(\alpha)$ given in (4.5) and (4.6), respectively, satisfy the relation $g_{2, T}(\alpha)=g_{1, T}(\alpha) r_{T}(\alpha)$ with $r_{T}(\alpha) \stackrel{\text { def }}{=}\left(\mathbf{a}^{H}(\alpha) \boldsymbol{\Pi}_{1, T} \mathbf{a}(\alpha)\right)+$ $\left|\mathbf{a}^{T}(\alpha) \boldsymbol{\Pi}_{2, T}^{H} \mathbf{a}(\alpha)\right|$, where in exact statistics $r\left(\theta_{k}\right) \neq 0$ (because

\footnotetext{
${ }^{4}$ These three algorithms have different behavior outside the asymptotic regime, as will be stressed in Section VI.
}

if $r\left(\theta_{k}\right)$ were to vanish, we would have $\mathbf{a}^{H}\left(\theta_{k}\right) \Pi_{1} \mathbf{a}\left(\theta_{k}\right)=0$ and $\left|\mathbf{a}^{H}\left(\theta_{k}\right) \boldsymbol{\Pi}_{2} \mathbf{a}^{*}\left(\theta_{k}\right)\right|=0$, and consequently

$$
\left(\begin{array}{c}
\mathbf{a}_{k} \\
\mathbf{a}_{k}^{*} e^{i \beta}
\end{array}\right)
$$

would belong to the signal space of $\mathbf{R}_{\tilde{y}}$ for all values of $\beta$, which leads to a contradiction with (4.4). Then, applying the proof [1, Theorem 3.2], the estimates minimizing $g_{1, T}$ and $g_{3, T}$ have the same asymptotic distribution and consequently algorithms 1 and 2 have the same asymptotic performances.

Then, to prove that algorithms 2 and 3 have the same asymptotic performances, we consider the first-order perturbation expansions of $\delta \theta_{k, T} \stackrel{\text { def }}{=} \theta_{k, T}-\theta_{k}$ as a function of $\delta \Pi_{1, T} \stackrel{\text { def }}{=}$ $\Pi_{1, T}-\Pi_{1}$ and $\delta \Pi_{2, T} \stackrel{\text { def }}{=} \Pi_{2, T}-\Pi_{2}$ given by these two algorithms. Following the lines of the derivation given in [13] where the standard MUSIC and root-MUSIC algorithms are replaced by algorithms 1 and 3, respectively, we prove in Appendix C that these algorithms satisfy the same perturbation expansion

$$
\begin{aligned}
\theta_{k, T}=\theta_{k} & +\mathbf{A}_{1, k} \operatorname{vec}\left(\delta \boldsymbol{\Pi}_{1, T}\right)+\mathbf{A}_{2, k} \operatorname{vec}\left(\delta \boldsymbol{\Pi}_{2, T}\right) \\
& +\mathbf{A}_{2, k}^{*} \operatorname{vec}\left(\delta \boldsymbol{\Pi}_{2, T}^{*}\right)+o\left(\delta \boldsymbol{\Pi}_{1, T}\right)+o\left(\delta \boldsymbol{\Pi}_{2, T}\right) .
\end{aligned}
$$

The proof is completed in Appendix C, where the DOA estimate given by algorithm 1 is proved to converge in distribution to a Gaussian distribution whose covariance matrice is given with (5.4), (5.5), and (5.6).

In case 2 , it is straightforward to prove the following theorem.

Theorem 6: The sequence $\sqrt{T}\left(\Theta_{T}-\Theta\right)$, where $\Theta_{T}$ is the DOA estimate given by the MUSIC-like algorithm (4.8) described in Section IV, converges in distribution to the zero-mean Gaussian distribution with covariance matrix

$$
\begin{aligned}
\left(\mathbf{C}_{\Theta}\right)_{k, l} & =\left(\mathbf{D}_{\Theta}^{\mathrm{Alg}_{4}} \mathbf{C}_{\Pi_{1}} \mathbf{D}_{\Theta}^{\mathrm{Alg}_{4}}\right)_{k, l} \\
& =\frac{2}{\alpha_{k} \alpha_{l}} \Re\left(\left(\mathbf{a}_{l}^{H} \mathbf{U}_{1} \mathbf{a}_{k}\right)\left(\mathbf{a}_{k}^{\prime H} \boldsymbol{\Pi} \mathbf{a}_{l}^{\prime}\right)\right)
\end{aligned}
$$

where $\mathbf{D}_{\Theta}^{\mathrm{Alg}_{4}}=\mathbf{D}_{\Theta}^{\mathrm{Alg}_{0}}$ is given in (A.3). Because

$$
\left(\begin{array}{ll}
\mathbf{U}_{1} & \mathbf{U}_{2} \\
\mathbf{U}_{2}^{*} & \mathbf{U}_{1}^{*}
\end{array}\right)=\sigma_{n}^{2} \tilde{\mathbf{S}}^{\#} \mathbf{R}_{\tilde{y}} \tilde{\mathbf{S}}^{\#}
$$

with $\tilde{\mathbf{S}}=\tilde{\mathbf{A}} \mathbf{R}_{\tilde{x}} \tilde{\mathbf{A}}^{H}$, we note that the performance of this algorithm is critical when $\mathbf{R}_{\tilde{x}}$ which interacts in $\tilde{\mathbf{S}}$ approaches singularity. This is particularly the case when the sources are uncorrelated with at least a noncircularity rate that tends to one (because in this case, $\operatorname{det}\left(\mathbf{R}_{\tilde{x}}\right)=\prod_{k=1}^{K}\left(\sigma_{k}^{4}\left(1-\rho_{k}^{2}\right)\right)$ ).

For a single source (5.8) gives

$$
C_{\theta_{1}}^{\mathrm{Alg}_{4}}=\frac{1}{\alpha_{1}\left(1-\rho_{1}^{2}\right)}\left[\frac{\sigma_{n}^{2}}{\sigma_{1}^{2}}+\frac{1}{\left\|\mathbf{a}_{1}\right\|^{2}} \frac{\left(1+\rho_{1}^{2}\right)}{\left(1-\rho_{1}^{2}\right)} \frac{\sigma_{n}^{4}}{\sigma_{1}^{4}}\right]
$$

and, consequently

$$
\begin{aligned}
C_{\theta_{1}}^{\mathrm{Alg}_{4}} & \geq C_{\theta_{1}}^{\mathrm{MUSIC}} \\
& =\frac{1}{\alpha_{1}}\left[\frac{\sigma_{n}^{2}}{\sigma_{1}^{2}}+\frac{1}{\left\|\mathbf{a}_{1}\right\|^{2}} \frac{\sigma_{n}^{4}}{\sigma_{1}^{4}}\right] \text { and } \\
\lim _{\rho_{1} \rightarrow 1} C_{\theta_{1}}^{\mathrm{Alg}_{4}} & =\infty .
\end{aligned}
$$

Thus, this algorithm is always outperformed by the standard MUSIC algorithm. This critical property will be studied for two sources, through numerical examples in Section VI.

Then considering the second algorithm proposed in case 2, we prove in Appendix D the following. 
Theorem 7: The sequence $\sqrt{T}\left(\Theta_{T}-\Theta\right)$, where $\Theta_{T}$ is the DOA estimate given by the weighted MUSIC algorithm introduced in Section IV converges in distribution to the zero-mean Gaussian distribution with covariance matrix

$$
\begin{aligned}
\left(\mathbf{C}_{\Theta}\right)_{k, l} & =\frac{1}{2 \alpha_{k} \alpha_{l}}\left(1 z^{*} z 1\right)\left(\left(\overline{\mathbf{A}}_{k}^{T} \tilde{\mathbf{U}}^{*} \overline{\mathbf{A}}_{l}^{*}\right) \otimes\left(\overline{\mathbf{A}}_{k}^{\prime H} \tilde{\mathbf{\Pi}} \overline{\mathbf{A}}_{l}^{\prime}\right)\right. \\
& \left.+\left(\overline{\mathbf{A}}_{k}^{\prime T} \tilde{\Pi}^{*} \overline{\mathbf{A}}_{l}^{*}\right) \otimes\left(\overline{\mathbf{A}}_{k}^{H} \tilde{\mathbf{U}} \overline{\mathbf{A}}_{l}\right)\right)\left(1 z^{*} z 1\right)^{H}
\end{aligned}
$$

with $z \stackrel{\text { def }}{=}\left(2 w_{1,2}^{*} / w_{1,1}+w_{2,2}\right), \quad \overline{\mathbf{A}}_{k} \stackrel{\text { def }}{=} \overline{\mathbf{A}}\left(\theta_{k}\right)$ and $\overline{\mathbf{A}}_{k}^{\prime} \stackrel{\text { def }}{=}\left(d \overline{\mathbf{A}}_{k} / d \theta_{k}\right)$. Furthermore, the value $z_{k}^{\text {opt }}$ that minimizes $\left(\mathbf{C}_{\Theta}\right)_{k, k}$ is given by

$$
z_{k}^{\mathrm{opt}}=-\frac{\mathbf{a}_{k}^{T} \mathbf{U}_{2}^{*} \mathbf{a}_{k}}{\mathbf{a}_{k}^{H} \mathbf{U}_{1} \mathbf{a}_{k}}
$$

for which the minimum value of $\left(\mathbf{C}_{\Theta}\right)_{k, k}$ is

$$
\min _{z}\left(\mathbf{C}_{\Theta}\right)_{k, k}=\frac{\operatorname{Det}\left(\overline{\mathbf{A}}_{k}^{H} \tilde{\mathbf{U}} \overline{\mathbf{A}}_{k}\right)}{2\left(\mathbf{a}_{k}^{H} \mathbf{U}_{1} \mathbf{a}_{k}\right)\left(\mathbf{a}_{k}^{\prime H} \Pi_{1} \mathbf{a}_{k}^{\prime}\right)} .
$$

For a single source, we prove in Appendix E.

Corollary 1: The asymptotic variance of the DOA estimate given by the optimal weighting MUSIC algorithm attains the noncircular Gaussian Cramer-Rao bound for all values of the noncircularity rate in the single source case.

Remark 1: The optimal value of the weight previously derived depends on the specific DOA whose variance is to be minimized, which means that the optimal weight is not the same for all DOAs. This, however, might have been expected as MUSIC estimates the DOAs one by one. In addition, it should be noted that $z_{k}^{\mathrm{opt}}$ is sample dependent. Consequently, this value ought to be replaced by a consistent estimate in the implementation of the optimal weighting MUSIC algorithm. This point will be described in Section VI). We note that this replacement of $z_{k}^{\text {opt }}$ by a consistent estimate has no effect on the asymptotic variance of the weighting MUSIC algorithm as it is proved in Appendix E.

Remark 2: For circular sources, $\mathbf{R}_{\tilde{y}}$ is block diagonal. This successively implies that $\tilde{\mathbf{S}}, \tilde{\mathbf{S}} \#$, and $\tilde{\mathbf{U}}$ are block diagonal. Consequently, $\mathbf{U}_{2}=\mathbf{O}, z_{k}^{\text {opt }}=0, \mathbf{W}_{\text {opt }}$ is diagonal, and the optimal weighting MUSIC algorithm reduces to the standard MUSIC algorithm. Then, (5.12) becomes $\min _{z}\left(\mathbf{C}_{\Theta}\right)_{k, k}=$ $\left(\mathbf{a}_{k}^{H} \mathbf{U} \mathbf{a}_{k} / 2 \mathbf{a}^{\prime}{ }_{k}^{H} \mathbf{\Pi}_{1} \mathbf{a}_{k}^{\prime}\right)$, which is the asymptotic variance given by (3.2).

To implement this optimal weighted MUSIC algorithm, we propose to use the following multistep procedure described in [11, sec. 7].

1. Determine standard MUSIC estimates of $\left(\theta_{k}\right)_{k=1, \ldots, K}$ from $\mathbf{R}_{y, T}$.

2. For $k=1, \ldots, K$, perform the following: Let $\theta_{k, T}^{0}$ denote the estimates obtained in step 1 . Use

$$
\left(\theta_{k, T}^{0}\right)_{k=1, \ldots, K}
$$

and the estimate $\mathbf{U}_{1, T}$ and $\mathbf{U}_{2, T}$ of $\mathbf{U}_{1}$ and $\mathbf{U}_{2}$ derived from $\tilde{\mathbf{R}}_{y, T}$ to obtain consistent estimates $z_{k, T}$ of $z_{k}^{\text {opt }}$. Then, determine improved estimates $\theta_{k, T}^{1}$ by locally minimizing the weighted MUSIC cost function (4.9) associated with $z_{k, T}$ around $\theta_{k, T}^{0}$.

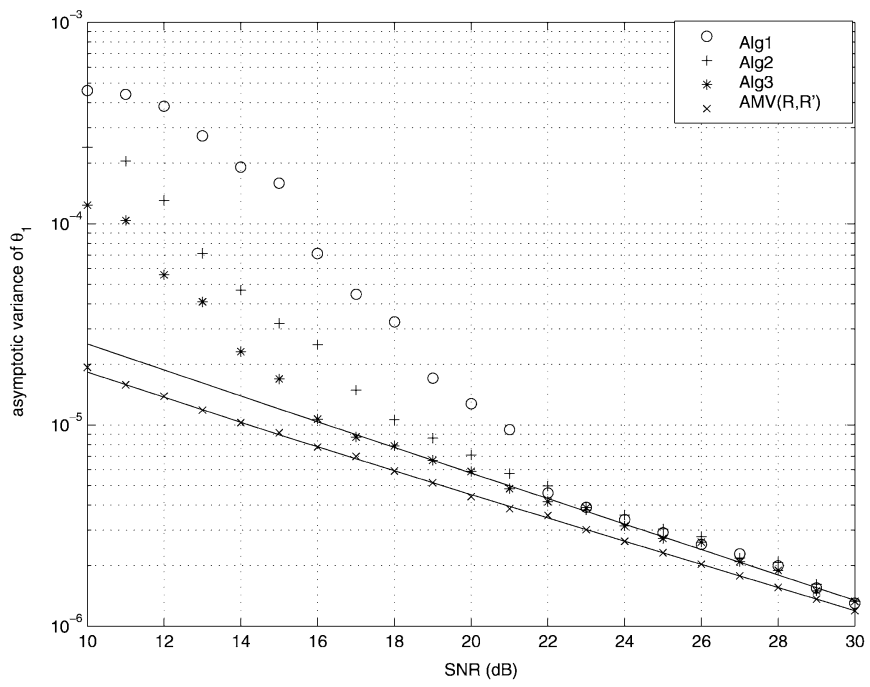

Fig. 1. Theoretical and empirical asymptotic variances given by algorithms 1, 2 , and 3 and AMV algorithm based on $\left(\mathbf{R}_{y, T}, \mathbf{R}_{y, T}^{\prime}\right)$ as a function of the SNR for $\Delta \theta=0.05 \mathrm{rad}, \Delta \phi=\pi 6 \mathrm{rad}$.

\section{ILLUSTRATIVE EXAMPLES}

In this section, we provide numerical illustrations and Monte Carlo simulations of the performance of the different algorithms presented in Section IV and numerical comparisons of the variances of these DOA estimates to the asymptotic variance of AMV estimators based on $\mathbf{R}_{\tilde{y}, T}$ (i.e., $\mathbf{R}_{y, T}$ and $\mathbf{R}_{y, T}^{\prime}$ ) and on $\mathbf{R}_{y, T}$ alone [3].

We consider throughout this section two uncorrelated ${ }^{5}$ equipowered (SNR $\left.\stackrel{\text { def }}{=}\left(\sigma_{1}^{2} / \sigma_{n}^{2}\right)\right)$ filtered or unfiltered BPSK modulated signals with identical noncircularity rate ( $\rho \stackrel{\text { def }}{=} \rho_{1}=\rho_{2}$ ) with phases of noncircularity $\phi_{1}$ and $\phi_{2}$. These signals impinge on a uniform linear array with $M=6$ sensors separated by a half-wavelength for which $\mathbf{a}_{k}=\left(1, e^{i \theta_{k}}, \ldots, e^{i(M-1) \theta_{k}}\right)^{T}$ where $\theta_{k}=\pi \sin \left(\alpha_{k}\right)$, with $\alpha_{k}$ the DOAs relative to the normal of array broadside. 1000 independent simulation runs have been performed to obtain the estimated variances and the number of snapshots is $T=500$ [respectively, $T=1000$ ] in case 1 [respectively, in case 2].

The first experiment illustrates Theorem 5 for which $\rho=1$. Figs. 1-3 exhibit the dependence of $\operatorname{var}\left(\theta_{1, T}\right)$ given by algorithms 1, 2, and 3, and by the AMV algorithm based on $\mathbf{R}_{\tilde{y}, T}$ (i.e., on $\mathbf{R}_{y, T}$ and $\mathbf{R}_{y, T}^{\prime}$ ), with the SNR, the DOA separation $\Delta \theta=\theta_{2}-\theta_{1}$, and the noncircularity phase separation $\Delta \phi=$ $\phi_{2}-\phi_{1}{ }^{6}$. Figs. 1 and 2 show that the domain of validity of our asymptotic analysis depends on the algorithm. Below an SNR threshold that is algorithm dependent, algorithm 3 (rootMUSIC-like algorithm) outperforms algorithm 2, which outperforms algorithm 1 , and naturally all three algorithms clearly outperform the standard MUSIC and the AMV algorithm based on $\mathbf{R}_{y, T}$ alone. In Fig. 2, we note that the asymptotic variances

${ }^{5} \mathrm{We}$ concentrate on uncorrelated sources because it was shown in [3] that expected benefits due to the noncircular property happens mainly for uncorrelated sources.

${ }^{6} \mathrm{By}$ virtue of numerical examples, the different theoretical variances depend on $\theta_{1}, \theta_{2}, \phi_{1}, \phi_{2}$ by only $\Delta \theta=\theta_{2}-\theta_{1}$ and $\Delta \phi=\phi_{2}-\phi_{1}$ in case 1 [only $\Delta \theta=\theta_{2}-\theta_{1}$ in case 2] for two equipowered sources with identical noncircularity rates. 


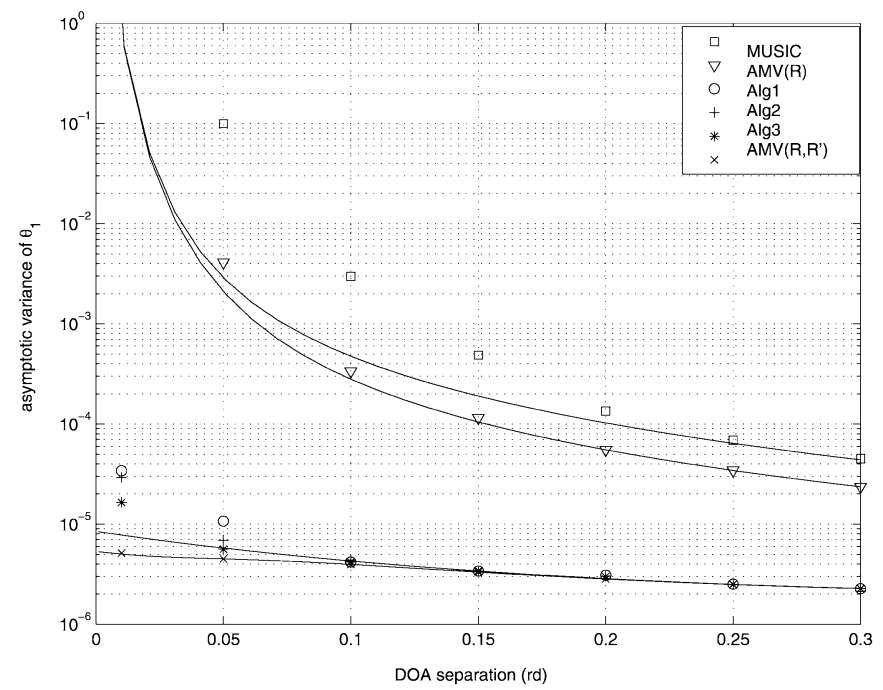

Fig. 2. Theoretical and empirical asymptotic variances given by algorithms $1,2,3$, standard MUSIC and AMV algorithms based on $\mathbf{R}_{y, T}$ only and on $\left(\mathbf{R}_{y, T}, \mathbf{R}_{y, T}^{\prime}\right)$ as a function of the DOA separation for SNR $=20 \mathrm{~dB}, \Delta \phi=\pi$ 6 rad.

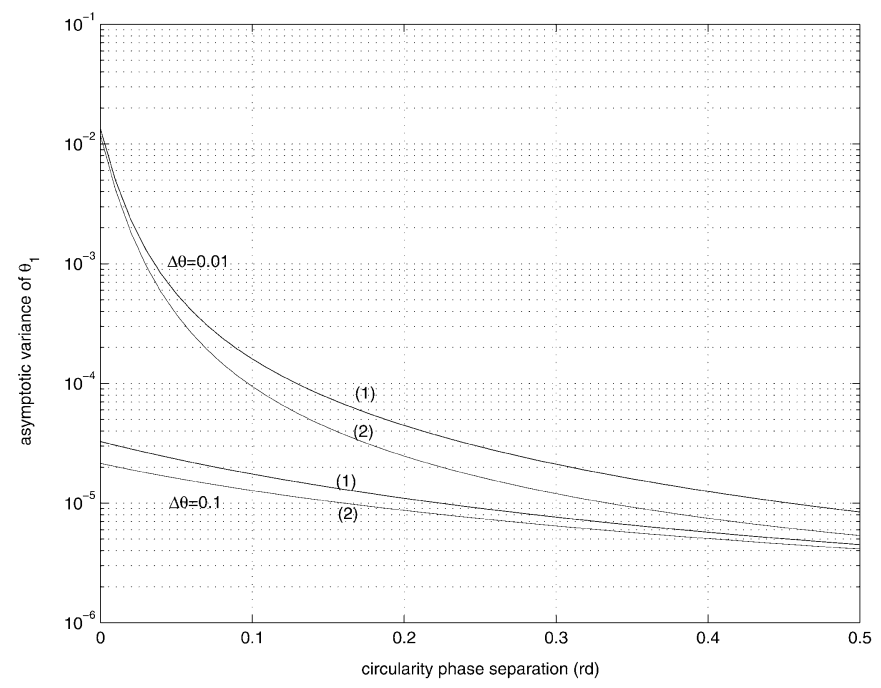

Fig. 3. Theoretical asymptotic variances given by algorithms 1,2 , and 3 and (1) by the AMV algorithm based on $\left(\mathbf{R}_{y, T}, \mathbf{R}_{y, T}^{\prime}\right)$ (2) as a function of the noncircularity phase separation for two DOA separations and SNR $=20 \mathrm{~dB}$.

given by algorithms 1, 2 and 3 and the AMV algorithm tend to a finite limit when the DOA separation decreases to zero. For algorithms 1, 2 and 3, this strange behavior is explained by the two nonzero eigenvalues $\left(\lambda_{k}\right)_{k=1,2}$ of $\tilde{\mathbf{S}}$ which interact in $\tilde{\mathbf{U}} \stackrel{\text { def }}{=} \sigma_{n}^{2} \tilde{\mathbf{S}}^{\#} \mathbf{R}_{\tilde{y}} \tilde{\mathbf{S}}^{\#}$ that appears in (5.5) of Theorem 5. With

$$
\begin{aligned}
\lambda_{k}=2 M \sigma_{1}^{2}\left(1+(-1)^{k} \cos (\right. & \left.(M-1) \frac{\Delta \theta}{2}-\Delta \phi\right) \\
& \left.\times \frac{\sin \left(M \frac{\Delta \theta}{2}\right)}{M \sin \left(\frac{\Delta \theta}{2}\right)}\right), \quad k=1,2
\end{aligned}
$$

we see that one of these eigenvalues approaches zero, and consequently the asymptotic variances increases without limit only if both $\Delta \theta$ and $\Delta \phi$ tend to zero. For the AMV algorithm, $\mathbf{C}_{\Theta}=$ $\left[\left(\mathcal{S}^{H} \mathbf{C}_{s}^{-1} \mathcal{S}\right)^{-1}\right]_{(1: K, 1: K)}$ (see the notations of [3]) and $\mathcal{S}$ is column rank deficient only if both $\Delta \theta$ and $\Delta \phi$ tend to zero

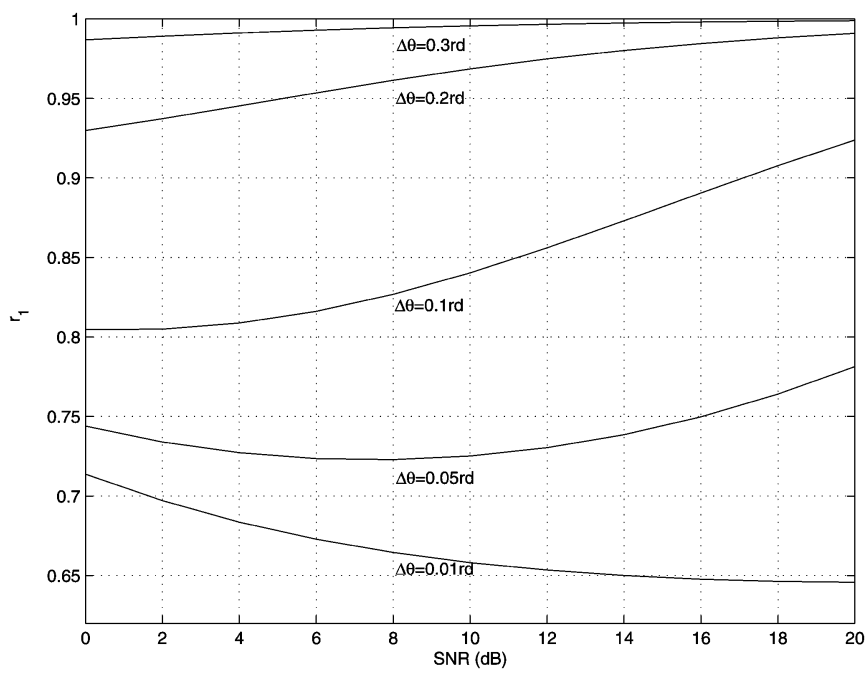

Fig. 4. Ratio $r_{1} \stackrel{\text { def }}{=} \operatorname{Var}_{\theta_{1}}^{\mathrm{AMV}\left(\mathrm{R}, \mathrm{R}^{\prime}\right)} / \operatorname{Var}_{\theta_{1}}^{\mathrm{Alg}_{1,2,3}}$ as a function of the SNR for different DOA separations, $\Delta \phi=\pi 6 \mathrm{rad}$.

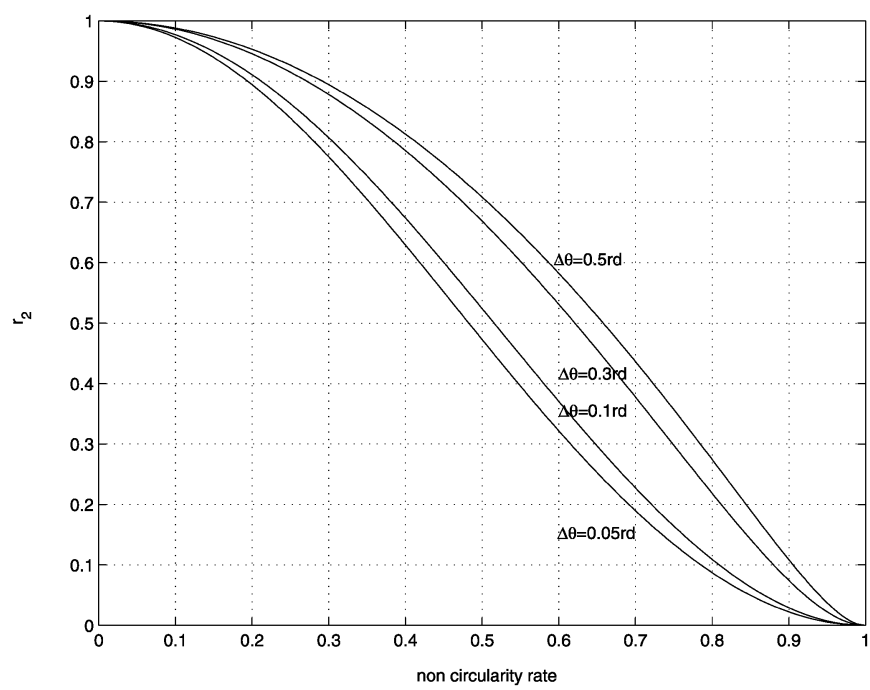

Fig. 5. Ratio $r_{2} \stackrel{\text { def }}{=} \operatorname{Var}_{\theta_{1}}^{\mathrm{MUSIC}(\mathrm{R})} / \operatorname{Var}_{\theta_{1}}^{\mathrm{Alg}_{4}}$ as a function of the noncircularity rate for different DOA separations for $\mathrm{SNR}=5 \mathrm{~dB}, \Delta \phi=\pi$ $6 \mathrm{rad}$.

as well. Fig. 3 illustrates the sensitivity of the performances to the noncircularity phase separation $\Delta \phi$, which is particularly prominent for low DOA separations. Figs. 1 and 2 show the good efficiency of these three algorithms compared to the AMV estimator based on $\mathbf{R}_{\tilde{y}, T}$, particularly for large DOA separations. To specify this point, Fig. 4 exhibits the ratio $r_{1} \stackrel{\text { def }}{=}$ $\operatorname{Var}_{\theta_{1}}^{\mathrm{AMV}\left(\mathrm{R}, \mathrm{R}^{\prime}\right)} / \operatorname{Var}_{\theta_{1}}^{\mathrm{Alg}_{1,2,3}}$ as a function of the SNR for different DOA separations. It shows that algorithms 1,2 , and 3 are very efficient, except for low DOA separations and low SNRs.

The second experiment considers arbitrary noncircularity rates $\rho$ (case 2). Fig. 5 exhibits the ratio $r_{2} \stackrel{\text { def }}{=}$ $\operatorname{Var}_{\theta_{1}}^{\mathrm{MUSIC}(\mathrm{R})} / \operatorname{Var}_{\theta_{1}}^{\mathrm{Alg}_{4}}$ as a function of the noncircularity rate for different DOA separations. It shows that algorithm 4 is worse than the standard MUSIC algorithm based on $\mathbf{R}_{y, T}$ alone, for all scenarios. This extends that a property proved by (5.9) in the single-source case.

In the following, we concentrate on the optimal weighted MUSIC algorithm (alg5) introduced in Section IV-B. Compared 


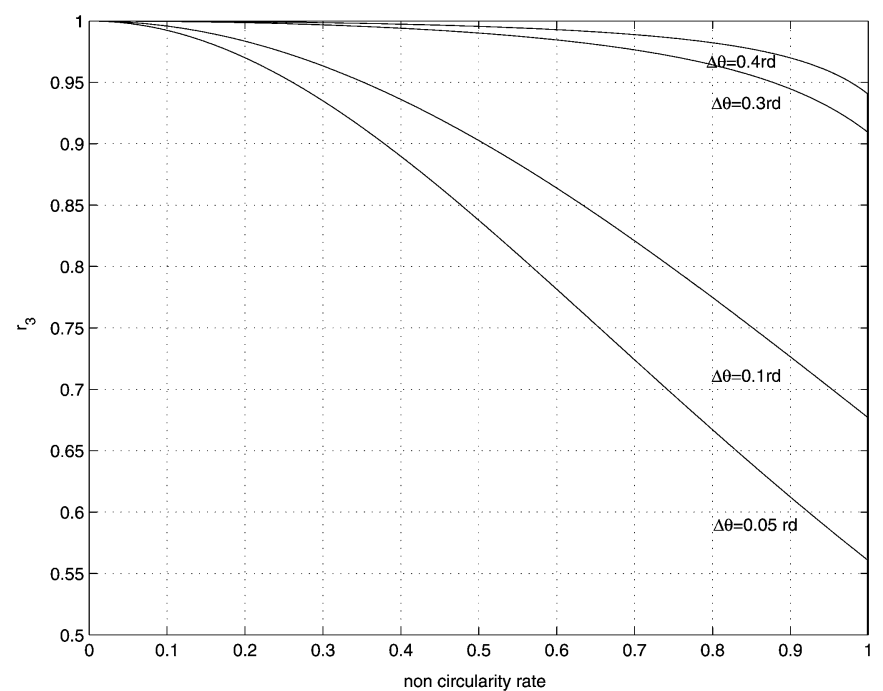

Fig. 6. Ratio $r_{3} \stackrel{\text { def }}{=} \operatorname{Var}_{\theta_{1}}^{\mathrm{Alg}} / \operatorname{Var}_{\theta_{1}}^{\mathrm{MUSIC}(\mathrm{R})}$ as a function of the noncircularity rate for different DOA separations for $\mathrm{SNR}=5 \mathrm{~dB}$.

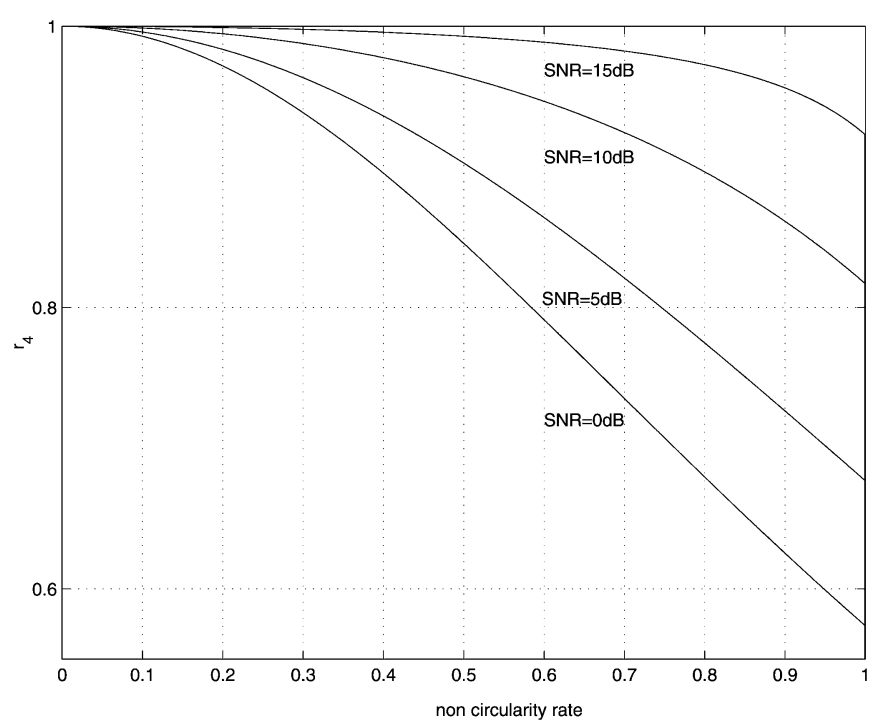

Fig. 7. Ratio $r_{4} \stackrel{\text { def }}{=} \operatorname{Var}_{\theta_{1}}^{\mathrm{Alg}} / \operatorname{Var}_{\theta_{1}}^{\mathrm{MUSIC}(\mathrm{R})}$ as a function of the noncircularity rate for different SNRs for $\Delta \theta=0.1 \mathrm{rad}$.

with the standard MUSIC algorithm based on $\mathbf{R}_{y, T}$, Figs. 6 and 7 show that algorithm 5 outperforms the standard MUSIC algorithm, particularly for low SNRs and DOA separations when the noncircularity rate $\rho$ increases.

The efficiency of this optimal weighted MUSIC algorithm is exhibited in Fig. 8 through the ratio $r_{4} \stackrel{\text { def }}{=}$ $\operatorname{Var}_{\theta_{1}}^{\mathrm{AMV}\left(\mathrm{R}, \mathrm{R}^{\prime}\right)} / \operatorname{Var}_{\theta_{1}}^{\mathrm{Alg} g_{5}}$. We show that, despite the fact that algorithm 5 improves the performance of the standard MUSIC algorithm based on $\mathbf{R}_{y, T}$ for low SNRs and DOA separations when the noncircularity rate $\rho$ increases, its efficiency decreases in these circumstances.

Tables I and II compare our theoretical asymptotic variance expressions with empirical mean square errors (MSEs) obtained

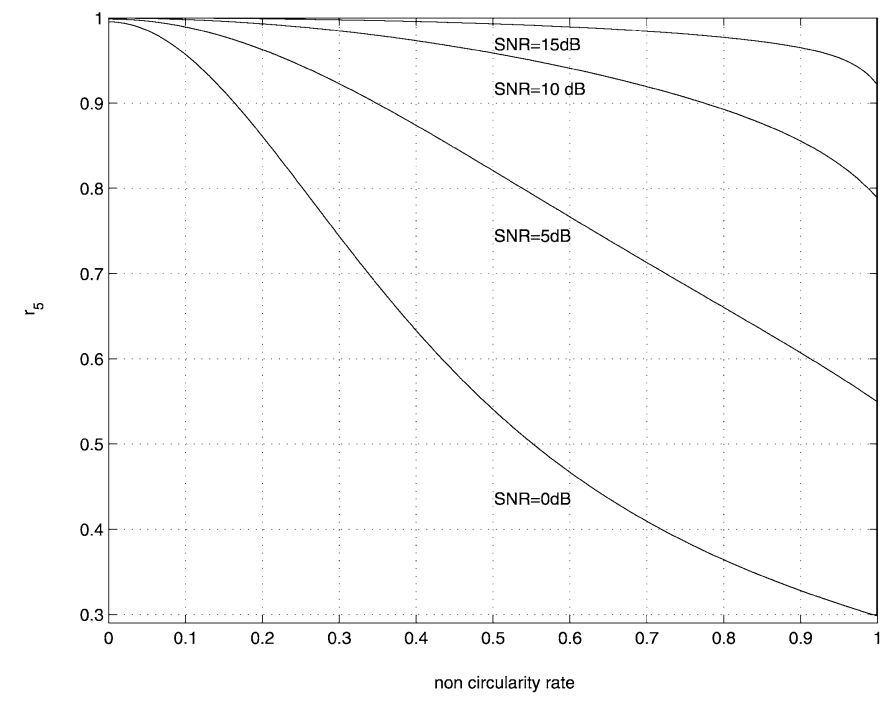

Fig. 8. Ratio $r_{5} \stackrel{\text { def }}{=} \operatorname{Var}_{\theta_{1}}^{\mathrm{AMV}\left(\mathrm{R}, \mathrm{R}^{\prime}\right)} / \operatorname{Var}_{\theta_{1}}^{\mathrm{Alg} g_{5}}$ as a function of the noncircularity rate for different SNRs for $\Delta \theta=0.1 \mathrm{rad}, \Delta \phi=\pi 6 \mathrm{rad}$.

from Monte Carlo simulations for the standard MUSIC and the optimal weighted MUSIC algorithms for $\rho=0.9, \Delta \theta=$ $0.2 \sim\{\mathrm{hbox}\{\operatorname{rad}\}\}$. We see that there is an agreement between the theoretical and empirical results beyond a SNR threshold. Below this threshold, the optimal weighted MUSIC algorithm largely outperforms the standard MUSIC algorithm.

\section{CONCLUSION}

This paper has provided a unifying framework to investigate the asymptotic performance of arbitrary subspace-based algorithms for estimating DOA's of narrowband complex noncircular sources by giving closed-form expressions of the covariance of the asymptotic distribution of extended projection matrices. Different robustness properties of the asymptotic covariance of the estimated DOA given by such algorithms are proved. These results are applied to different MUSIC-like algorithms. We have proved that such specific algorithms largely outperform the standard MUSIC algorithm in the case of uncorrelated sources with maximum noncircularity rate. In the general case of nonsingular extended spatial covariance of the sources, the optimal weighted MUSIC that we have introduced outperforms the standard MUSIC algorithm as well, but the offered performance gain is noticeable for low SNRs and DOA separations only. Furthermore, this optimal weighted MUSIC is computationally more demanding than the standard MUSIC algorithm. Consequently, from an application viewpoint, this gain in performance may not motivate the extra computational complexity. In this general case of nonsingular extended spatial covariance of the sources, only multidimensional nonlinear optimization algorithms such as the subspace-based AMV estimator seems to be able to totally benefit of the noncircular property. A study to deal with this issue is underway. 
TABLE I

$\Delta \theta=0.2 \mathrm{rad}$

\begin{tabular}{|c||c|c||c|c|}
\hline \multicolumn{1}{|c||}{} & \multicolumn{2}{c||}{ Standard MUSIC } & \multicolumn{2}{c||}{ Optimal weighted MUSIC } \\
\hline SNR (dB) & empirical MSE & theoretical variance & empirical MSE & theoretical variance \\
\hline 6 & $4.452 .10^{-3}$ & $4.589 .10^{-4}$ & $3.154 .10^{-4}$ & $4.151 .10^{-4}$ \\
\hline 8 & $1.600 .10^{-3}$ & $2.604 .10^{-4}$ & $2.344 .10^{-4}$ & $2.449 .10^{-4}$ \\
\hline 10 & $2.899 .10^{-4}$ & $1.527 .10^{-4}$ & $1.561 .10^{-4}$ & $1.474 .10^{-4}$ \\
\hline 20 & $1.338 .10^{-5}$ & $1.348 .10^{-5}$ & $1.337 .10^{-5}$ & $1.347 .10^{-5}$ \\
\hline
\end{tabular}

TABLE II

$\Delta \theta=0.2 \mathrm{rad}$ AND SNR $=8 \mathrm{~dB}$

\begin{tabular}{|c||c|c||c|c||}
\hline \multicolumn{1}{|c||}{} & \multicolumn{2}{c||}{ Standard MUSIC } & \multicolumn{2}{c||}{ Optimal weighted MUSIC } \\
\hline & empirical MSE & theoretical variance & empirical MSE & theoretical variance \\
\hline$\theta_{1}$ & $1.600 .10^{-3}$ & $2.604 .10^{-4}$ & $2.344 .10^{-4}$ & $2.449 .10^{-4}$ \\
\hline$\theta_{2}$ & $1.800 .10^{-3}$ & $2.604 .10^{-4}$ & $2.457 .10^{-4}$ & $2.449 .10^{-4}$ \\
\hline
\end{tabular}

APPENDIX A

\section{PROOF OF THEOREM 1}

Because $\Pi_{T}^{\prime}$ is the orthogonal projector onto the noise subspace of the Hermitian matrix $\mathbf{R}_{y, T}^{\prime} \mathbf{R}_{y, T}^{\prime H}$, the standard perturbation result (B.1) for orthogonal projectors associated with invariant subspaces of Hermitian matrices can be applied, as follows:

$$
\begin{aligned}
\delta\left(\boldsymbol{\Pi}^{\prime}\right)= & -\boldsymbol{\Pi}^{\prime} \delta\left(\mathbf{R}_{y}^{\prime} \mathbf{R}_{y}^{\prime H}\right)\left(\mathbf{R}_{y}^{\prime} \mathbf{R}_{y}^{\prime H}\right)^{\#} \\
& -\left(\mathbf{R}_{y}^{\prime} \mathbf{R}_{y}^{\prime H}\right)^{\#} \delta\left(\mathbf{R}_{y}^{\prime} \mathbf{R}_{y}^{\prime H}\right) \boldsymbol{\Pi}^{\prime}+o\left(\delta\left(\mathbf{R}_{y}^{\prime} \mathbf{R}_{y}^{\prime H}\right)\right) .
\end{aligned}
$$

Using $\delta\left(\mathbf{R}_{y}^{\prime} \mathbf{R}_{y}^{\prime H}\right)=\delta\left(\mathbf{R}_{y}^{\prime}\right) \mathbf{R}_{y}^{\prime H}+\mathbf{R}_{y}^{\prime} \delta\left(\mathbf{R}_{y}^{\prime H}\right)+o\left(\delta\left(\mathbf{R}_{y}^{\prime}\right)\right)$, $\boldsymbol{\Pi}^{\prime} \mathbf{R}_{y}^{\prime}=\mathbf{O}$, and $\mathbf{R}_{y}^{\prime H}\left(\mathbf{R}_{y}^{\prime} \mathbf{R}_{y}^{\prime H}\right)^{\#}=\mathbf{R}_{y}^{\prime \#}$, we obtain

$$
\delta\left(\boldsymbol{\Pi}^{\prime}\right)=-\Pi^{\prime} \delta\left(\mathbf{R}_{y}^{\prime}\right) \mathbf{R}_{y}^{\prime \#}-\mathbf{R}_{y}^{\prime H} \delta\left(\mathbf{R}_{y}^{\prime H}\right) \boldsymbol{\Pi}^{\prime}+o\left(\delta\left(\mathbf{R}_{y}^{\prime}\right)\right) .
$$

Then using the standard theorem of continuity (see, e.g., [18, p. 122]) on regular functions of asymptotically Gaussian statistics, the asymptotic behaviors of $\boldsymbol{\Pi}_{T}^{\prime}$ and $\mathbf{R}_{y, T}^{\prime}$ are directly related and the first covariance matrix of the asymptotically Gaussian distribution of $\boldsymbol{\Pi}_{T}^{\prime}$ can be written as

$$
\begin{aligned}
& \mathbf{C}_{\Pi^{\prime}}=\left(\mathbf{R}_{y}^{\prime \#} \otimes \boldsymbol{\Pi}^{\prime} \quad \boldsymbol{\Pi}^{\prime *} \otimes \mathbf{R}_{y}^{\prime * \#}\right) \\
& \times\left(\begin{array}{cc}
\mathbf{C}_{r_{y}^{\prime}} & \mathbf{C}_{r_{y}^{\prime}}^{\prime} \\
\mathbf{C}_{r_{y}^{\prime}}^{\prime} * & \mathbf{C}_{r_{y}^{\prime}}^{*}
\end{array}\right)\left(\begin{array}{l}
\mathbf{R}_{y}^{\prime *} \otimes \Pi^{\prime} \\
\Pi^{\prime *} \otimes \mathbf{R}_{y}^{\prime \#}
\end{array}\right)
\end{aligned}
$$

where

$$
\text { the }
$$

$$
\text { expressions }
$$

of $\left.\mathbf{C}_{r_{y}^{\prime}}=E\left(\mathbf{y}_{t} \otimes \mathbf{y}_{t}-\operatorname{vec}\left(\mathbf{R}_{y}^{\prime}\right)\right)\left(\mathbf{y}_{t} \otimes \mathbf{y}_{t}-\operatorname{vec}\left(\mathbf{R}_{y}^{\prime}\right)\right)^{H}\right)$ and $\left.\mathbf{C}_{r_{y}^{\prime}}^{\prime \prime}=E\left(\mathbf{y}_{t} \otimes \mathbf{y}_{t}-\operatorname{vec}\left(\mathbf{R}_{y}^{\prime}\right)\right)\left(\mathbf{y}_{t} \otimes \mathbf{y}_{t}-\operatorname{vec}\left(\mathbf{R}_{y}^{\prime}\right)\right)^{T}\right)$ are given in [3, Lemma 2]. Inserting these expressions in (A.1) and using $\Pi^{\prime} \mathbf{A}=\mathbf{O}, \mathbf{R}_{y}^{\prime \#} \mathbf{R}_{y} \Pi^{\prime}=\mathbf{O}$, we obtain after tedious but simple algebra manipulations

$$
\mathbf{C}_{\Pi^{\prime}}=\Pi^{\prime *} \otimes \mathbf{U}+\mathbf{U}^{*} \otimes \boldsymbol{\Pi}^{\prime}
$$

where $\mathbf{U}$ is given here by $\sigma_{n}^{2} \mathbf{S}^{* \#} \mathbf{R}_{y}^{T} \mathbf{S}^{\prime \#}$. Using again the standard theorem of continuity, the DOAs estimated by the MUSIC algorithm based on $\Pi_{T}^{\prime}$ are asymptotically Gaussian distributed with covariance $\mathbf{C}_{\Theta}=\mathbf{D}_{\Theta}^{\mathrm{Alg}{ }_{0}} \mathbf{C}_{\Pi^{\prime}}\left(\mathbf{D}_{\Theta}^{\mathrm{Alg}}\right)^{H}$, where the Jacobian matrix $\mathbf{D}_{\Theta}^{\mathrm{Alg} 0}$ of the mapping that associates $\Theta_{T}$ to $\boldsymbol{\Pi}_{T}^{\prime}$ is given by

$$
\mathbf{D}_{\Theta}^{A l_{0}}=\left(\begin{array}{c}
\mathbf{d}_{1}^{T} \\
\vdots \\
\mathbf{d}_{K}^{T}
\end{array}\right) \text { with } \mathbf{d}_{k}^{T}=\frac{-1}{\alpha_{k}}\left(\mathbf{a}_{k}^{\prime T} \otimes \mathbf{a}_{k}^{H}+\mathbf{a}_{k}^{T} \otimes \mathbf{a}_{k}^{\prime H}\right)
$$

straightforwardly obtained from a first-order expansion of $\left(\partial g_{0, T}(\theta) / \partial \theta\right)_{\mid \theta=\theta_{k}+\delta \theta_{k, T}}=0$. Using expression (A.2) of $\mathbf{C}_{\Pi^{\prime}}$, expression (3.2) of $\mathbf{C}_{\Theta}$ is straightforwardly deduced.

In the case of a single source, $\mathbf{U}=$ $\left(\sigma_{n}^{2}\left(\sigma_{1}^{2}\left\|\mathbf{a}_{1}\right\|^{2}+\sigma_{n}^{2}\right) / \sigma_{1}^{4} \rho_{1}^{2}\left\|\mathbf{a}_{1}\right\|^{6}\right) \mathbf{a}_{1} \mathbf{a}_{1}^{H}, \quad$ and the expression of $C_{\theta_{1}}$ follows.

\section{APPENDIX B}

\section{PROOF OF THEOREM 3}

The proof relies on the standard central limit theorem applied to the independent equidistributed complex noncircular random variables $\tilde{\mathbf{y}}_{t}^{*} \otimes \tilde{\mathbf{y}}_{t}$ with $\tilde{\mathbf{y}}_{t}=\tilde{\mathbf{A}} \tilde{\mathbf{x}}_{t}+\tilde{\mathbf{n}}_{t}$. Thanks to simple algebraic manipulations of $\mathbf{C}_{r_{\tilde{y}}}=$ $E\left(\left(\tilde{\mathbf{y}}_{t}^{*} \otimes \tilde{\mathbf{y}}_{t}-\operatorname{vec}\left(\mathbf{R}_{\tilde{y}}\right)\right)\left(\tilde{\mathbf{y}}_{t}^{*} \otimes \tilde{\mathbf{y}}_{t}-\operatorname{vec}\left(\mathbf{R}_{\tilde{y}}\right)\right)^{H}\right), \quad$ we straightforwardly obtain

$\mathbf{C}_{r_{\tilde{y}}}=\mathbf{R}_{\tilde{y}}^{*} \otimes \mathbf{R}_{\tilde{y}}+\mathbf{K}_{2 M}\left(\mathbf{R}_{\tilde{y}}^{\prime} \otimes \mathbf{R}_{\tilde{y}}^{\prime *}\right)$

$$
+\left(\tilde{\mathbf{A}}^{*} \otimes \tilde{\mathbf{A}}\right) \mathbf{Q}_{\tilde{x}}\left(\tilde{\mathbf{A}}^{T} \otimes \tilde{\mathbf{A}}^{H}\right)
$$

with $\mathbf{R}_{\tilde{y}}^{\prime} \stackrel{\text { def }}{=} E\left(\tilde{\mathbf{y}}_{t} \tilde{\mathbf{y}}_{t}^{T}\right)$ and where $\left(\mathbf{Q}_{\tilde{x}}\right)_{i+(j-1) 2 K, k+(l-1) 2 K}=$ $\operatorname{Cum}\left(\left(\tilde{\mathbf{x}}_{t}\right)_{i},\left(\tilde{\mathbf{x}}_{t}\right)_{j}^{*},\left(\tilde{\mathbf{x}}_{t}\right)_{k}^{*},\left(\tilde{\mathbf{x}}_{t}\right)_{l}\right)$. Then using the standard perturbation result for orthogonal projectors [17] (see also [13]) applied to $\tilde{\Pi}$ associated with the noise subspace of $\mathbf{R}_{\tilde{y}}$

$$
\delta(\tilde{\boldsymbol{\Pi}})=-\tilde{\boldsymbol{\Pi}} \delta\left(\mathbf{R}_{\tilde{y}}\right) \tilde{\mathbf{S}}^{\#}-\tilde{\mathbf{S}}^{\#} \delta\left(\mathbf{R}_{\tilde{y}}\right) \tilde{\boldsymbol{\Pi}}+o\left(\delta\left(\mathbf{R}_{\tilde{y}}\right)\right)
$$

the asymptotic behaviors of $\tilde{\Pi}_{T}$ and $\mathbf{R}_{\tilde{y}, T}$ are directly related. The standard theorem (see, e.g., [18, p. 122]) on regular functions of asymptotically Gaussian statistics applies and the first 
covariance matrix of the asymptotically Gaussian distribution of $\tilde{\Pi}_{T}$ can be written as

$$
\begin{aligned}
\mathbf{C}_{\tilde{\Pi}}= & \left(\left(\tilde{\boldsymbol{\Pi}}^{*} \otimes \tilde{\mathbf{S}}^{\#}\right)+\left(\tilde{\mathbf{S}}^{\#} \otimes \tilde{\boldsymbol{\Pi}}\right)\right) \\
& \times \mathbf{C}_{r_{\tilde{y}}}\left(\left(\tilde{\boldsymbol{\Pi}}^{*} \otimes \tilde{\mathbf{S}}^{\#}\right)+\left(\tilde{\mathbf{S}} \#^{*} \otimes \tilde{\mathbf{\Pi}}\right)\right) \\
= & \left(\left(\tilde{\boldsymbol{\Pi}}^{*} \otimes \tilde{\mathbf{S}}^{\#}\right)+\left(\tilde{\mathbf{S}}^{\#} \otimes \tilde{\boldsymbol{\Pi}}\right)\right) \\
& \times\left(\mathbf{R}_{\tilde{y}}^{*} \otimes \mathbf{R}_{\tilde{y}}+\mathbf{K}_{2 M}\left(\mathbf{R}_{\tilde{y}}^{\prime} \otimes \mathbf{R}_{\tilde{y}}^{\prime *}\right)\right) \\
& \times\left(\left(\tilde{\boldsymbol{\Pi}}^{*} \otimes \tilde{\mathbf{S}}^{\#}\right)+\left(\tilde{\mathbf{S}} \#^{*} \otimes \tilde{\mathbf{\Pi}}\right)\right) \\
= & \left(\mathbf{I}_{4 M^{2}}+\mathbf{K}_{2 M}\left(\mathbf{J}_{M} \otimes \mathbf{J}_{M}\right)\right) \\
& \times\left(\left(\tilde{\boldsymbol{\Pi}}^{*} \otimes \tilde{\mathbf{U}}\right)+\left(\tilde{\mathbf{U}}^{*} \otimes \tilde{\mathbf{\Pi}}\right)\right)
\end{aligned}
$$

where $\tilde{\boldsymbol{\Pi}} \tilde{\mathbf{A}}=\mathbf{O}$, and $\mathbf{R}_{\tilde{y}}^{*}=\mathbf{J}_{M} \mathbf{R}_{\tilde{y}}$ and $\tilde{\mathbf{S}}{ }^{\#} \mathbf{R}_{\tilde{y}} \tilde{\boldsymbol{\Pi}}=\mathbf{O}$ are used in the second and third equalities, respectively.

Proving the convergence in distribution of the second statistic follows the same lines where the terms of

$$
\begin{aligned}
& \mathbf{C}_{\Pi_{1}, \Pi_{2}, \Pi_{2}^{*}} \\
& =\lim _{T \rightarrow \infty} \frac{1}{T} E\left[\left(\begin{array}{c}
\operatorname{vec}\left(\boldsymbol{\Pi}_{1, T}-\boldsymbol{\Pi}_{1}\right) \\
\operatorname{vec}\left(\boldsymbol{\Pi}_{2, T}-\boldsymbol{\Pi}_{2}\right) \\
\operatorname{vec}\left(\boldsymbol{\Pi}_{2, T}^{*}-\boldsymbol{\Pi}_{2}^{*}\right)
\end{array}\right)\left(\begin{array}{c}
\operatorname{vec}\left(\boldsymbol{\Pi}_{1, T}-\boldsymbol{\Pi}_{1}\right) \\
\operatorname{vec}\left(\boldsymbol{\Pi}_{2, T}-\boldsymbol{\Pi}_{2}\right) \\
\operatorname{vec}\left(\boldsymbol{\Pi}_{2, T}^{*}-\boldsymbol{\Pi}_{2}^{*}\right)
\end{array}\right)^{H}\right] \\
& =\left(\begin{array}{ccc}
\mathbf{C}_{\Pi_{1}} & \mathbf{C}_{\Pi_{2}, \Pi_{1}}^{H} & \mathbf{C}_{\Pi_{2}^{*}, \Pi_{1}}^{H} \\
\mathbf{C}_{\Pi_{2}, \Pi_{1}} & \mathbf{C}_{\Pi_{2}} & \mathbf{C}_{\Pi_{2}^{*}, \Pi_{2}}^{H} \\
\mathbf{C}_{\Pi_{2}^{*}, \Pi_{1}} & \mathbf{C}_{\Pi_{2}^{*}, \Pi_{2}} & \mathbf{C}_{\Pi_{2}^{*}}^{*}
\end{array}\right)
\end{aligned}
$$

can be deduced from the expression of

$$
\begin{aligned}
& \mathbf{C}_{\tilde{\Pi}}=\lim _{T \rightarrow \infty} \frac{1}{T} E\left[\operatorname{vec}\left(\begin{array}{ll}
\Pi_{1, T}-\Pi_{1} & \Pi_{2, T}-\Pi_{2} \\
\Pi_{2, T}^{*}-\Pi_{2}^{*} & \Pi_{1, T}^{*}-\Pi_{1}^{*}
\end{array}\right)\right. \\
& \left.\times \operatorname{vec}^{H}\left(\begin{array}{ll}
\Pi_{1, T}-\Pi_{1} & \Pi_{2, T}-\Pi_{2} \\
\Pi_{2, T}^{*}-\Pi_{2}^{*} & \Pi_{1, T}^{*}-\Pi_{1}^{*}
\end{array}\right)\right] \\
& =\left(\begin{array}{ll}
\mathbf{G} & \mathbf{O} \\
\mathbf{O} & \mathbf{G}
\end{array}\right) \lim _{T \rightarrow \infty} \frac{1}{T} \\
& \times E\left(\left[\begin{array}{c}
\operatorname{vec}\left(\boldsymbol{\Pi}_{1, T}-\boldsymbol{\Pi}_{1}\right) \\
\operatorname{vec}\left(\boldsymbol{\Pi}_{2, T}^{*}-\boldsymbol{\Pi}_{2}^{*}\right) \\
\operatorname{vec}\left(\boldsymbol{\Pi}_{2, T}-\boldsymbol{\Pi}_{2}\right) \\
\operatorname{vec}\left(\boldsymbol{\Pi}_{1, T}^{*}-\boldsymbol{\Pi}_{1}^{*}\right)
\end{array}\right]\left[\begin{array}{c}
\operatorname{vec}\left(\boldsymbol{\Pi}_{1, T}-\boldsymbol{\Pi}_{1}\right) \\
\operatorname{vec}\left(\boldsymbol{\Pi}_{2, T}^{*}-\boldsymbol{\Pi}_{2}^{*}\right) \\
\operatorname{vec}\left(\boldsymbol{\Pi}_{2, T}-\boldsymbol{\Pi}_{2}\right) \\
\operatorname{vec}\left(\boldsymbol{\Pi}_{1, T}^{*}-\boldsymbol{\Pi}_{1}^{*}\right)
\end{array}\right]^{H}\right) \\
& \times\left(\begin{array}{cc}
\mathbf{G}^{T} & \mathbf{O} \\
\mathbf{O} & \mathbf{G}^{T}
\end{array}\right) \\
& =\left(\begin{array}{cc}
\mathbf{G} & \mathbf{O} \\
\mathbf{O} & \mathbf{G}
\end{array}\right)\left(\begin{array}{cccc}
\mathbf{C}_{\Pi_{1}} & \mathbf{C}_{\Pi_{2}^{*}, \Pi_{1}}^{H} & \mathbf{C}_{\Pi_{2}, \Pi_{1}}^{H} & \mathbf{C}_{\Pi_{1}, \Pi_{1}^{*}} \\
\mathbf{C}_{\Pi_{2}^{*}, \Pi_{1}} & \mathbf{C}_{\Pi_{2}^{*}} & \mathbf{C}_{\Pi_{2}^{*}, \Pi_{2}} & \mathbf{C}_{\Pi_{2}, \Pi_{1}}^{*} \\
\mathbf{C}_{\Pi_{2}, \Pi_{1}} & \mathbf{C}_{\Pi_{2}^{*}, \Pi_{2}}^{H} & \mathbf{C}_{\Pi_{2}} & \mathbf{C}_{\Pi_{2}^{*}, \Pi_{1}}^{*} \\
\mathbf{C}_{\Pi_{1}, \Pi_{1}^{*}}^{H} & \mathbf{C}_{\Pi_{2}, \Pi_{1}}^{T} & \mathbf{C}_{\Pi_{2}^{*}, \Pi_{1}}^{T} & \mathbf{C}_{\Pi_{1}^{*}}^{*}
\end{array}\right) \\
& \times\left(\begin{array}{cc}
\mathbf{G}^{T} & \mathbf{O} \\
\mathbf{O} & \mathbf{G}^{T}
\end{array}\right)
\end{aligned}
$$

where $\mathbf{G}$ is the block permutation matrix defined by

$$
\operatorname{vec}\left(\begin{array}{l}
\mathbf{A} \\
\mathbf{B}
\end{array}\right)=\mathbf{G}\left(\begin{array}{c}
\operatorname{vec}(\mathbf{A}) \\
\operatorname{vec}(\mathbf{B})
\end{array}\right)
$$

With expressions (B.3) and (5.1) of $\tilde{\Pi}$, we obtain

$$
\begin{aligned}
& \left(\begin{array}{cccc}
\mathbf{C}_{\Pi_{1}} & \mathbf{C}_{\Pi_{2}^{*}, \Pi_{1}}^{H} & \mathbf{C}_{\Pi_{2}, \Pi_{1}}^{H} & \mathbf{C}_{\Pi_{1}, \Pi_{1}^{*}} \\
\mathbf{C}_{\Pi_{2}^{*}, \Pi_{1}} & \mathbf{C}_{\Pi_{2}^{*}} & \mathbf{C}_{\Pi_{2}^{*}, \Pi_{2}} & \mathbf{C}_{\Pi_{2}, \Pi_{1}}^{*} \\
\mathbf{C}_{\Pi_{2}, \Pi_{1}} & \mathbf{C}_{\Pi_{2}^{*}, \Pi_{2}}^{H} & \mathbf{C}_{\Pi_{2}} & \mathbf{C}_{\Pi_{2}^{*}, \Pi_{1}}^{*} \\
\mathbf{C}_{\Pi_{1}, \Pi_{1}^{*}}^{H} & \mathbf{C}_{\Pi_{2}, \Pi_{1}}^{T} & \mathbf{C}_{\Pi_{2}^{*}, \Pi_{1}}^{T} & \mathbf{C}_{\Pi_{1}^{*}}
\end{array}\right) \\
& =\left(\begin{array}{cc}
\mathbf{G}^{T} & \mathbf{O} \\
\mathbf{O} & \mathbf{G}^{T}
\end{array}\right) \\
& \times\left(\left[\begin{array}{ll}
\boldsymbol{\Pi}_{1}^{*} & \boldsymbol{\Pi}_{2}^{*} \\
\boldsymbol{\Pi}_{2} & \boldsymbol{\Pi}_{1}
\end{array}\right] \otimes\left[\begin{array}{ll}
\mathbf{U}_{1} & \mathbf{U}_{2} \\
\mathbf{U}_{2}^{*} & \mathbf{U}_{1}^{*}
\end{array}\right]+\left[\begin{array}{ll}
\mathbf{U}_{1}^{*} & \mathbf{U}_{2}^{*} \\
\mathbf{U}_{2} & \mathbf{U}_{1}
\end{array}\right] \otimes\left[\begin{array}{ll}
\boldsymbol{\Pi}_{1} & \boldsymbol{\Pi}_{2} \\
\boldsymbol{\Pi}_{2}^{*} & \boldsymbol{\Pi}_{1}^{*}
\end{array}\right]\right. \\
& +\mathbf{K}_{2 M}\left(\left[\begin{array}{ll}
\Pi_{2} & \boldsymbol{\Pi}_{1} \\
\boldsymbol{\Pi}_{1}^{*} & \boldsymbol{\Pi}_{2}^{*}
\end{array}\right] \otimes\left[\begin{array}{ll}
\mathbf{U}_{2}^{*} & \mathbf{U}_{1}^{*} \\
\mathbf{U}_{1} & \mathbf{U}_{2}
\end{array}\right]\right) \\
& \left.+\mathbf{K}_{2 M}\left(\left[\begin{array}{ll}
\mathbf{U}_{2} & \mathbf{U}_{1} \\
\mathbf{U}_{1}^{*} & \mathbf{U}_{2}^{*}
\end{array}\right] \otimes\left[\begin{array}{ll}
\boldsymbol{\Pi}_{2}^{*} & \boldsymbol{\Pi}_{1}^{*} \\
\boldsymbol{\Pi}_{1} & \boldsymbol{\Pi}_{2}
\end{array}\right]\right)\right)\left(\begin{array}{ll}
\mathbf{G} & \mathbf{O} \\
\mathbf{O} & \mathbf{G}
\end{array}\right) .
\end{aligned}
$$

Then, using the following two identities deduced from the definition of the permutation matrices $\mathbf{G}$ and $\mathbf{K}_{2 M}$ for any $M \times M$ matrices $\mathbf{A}, \mathbf{B}, \mathbf{C}$, and $\mathbf{D}$

$$
\begin{aligned}
& \left(\begin{array}{cc}
\mathbf{G}^{T} & \mathbf{O} \\
\mathbf{O} & \mathbf{G}^{T}
\end{array}\right)\left(\left[\begin{array}{cc}
\mathbf{A} & \mathbf{B} \\
\mathbf{B}^{*} & \mathbf{A}^{*}
\end{array}\right] \otimes\left[\begin{array}{cc}
\mathbf{C} & \mathbf{D} \\
\mathbf{D}^{*} & \mathbf{C}^{*}
\end{array}\right]\right)\left(\begin{array}{cc}
\mathbf{G} & \mathbf{O} \\
\mathbf{O} & \mathbf{G}
\end{array}\right) \\
& =\left(\begin{array}{cccc}
\mathbf{A} \otimes \mathbf{C} & \mathbf{A} \otimes \mathbf{D} & \mathbf{B} \otimes \mathbf{C} & \mathbf{B} \otimes \mathbf{D} \\
\mathbf{A} \otimes \mathbf{D}^{*} & \mathbf{A} \otimes \mathbf{C}^{*} & \mathbf{B} \otimes \mathbf{D}^{*} & \mathbf{B} \otimes \mathbf{C}^{*} \\
\mathbf{B}^{*} \otimes \mathbf{C} & \mathbf{B}^{*} \otimes \mathbf{D} & \mathbf{A}^{*} \otimes \mathbf{C} & \mathbf{A}^{*} \otimes \mathbf{D} \\
\mathbf{B}^{*} \otimes \mathbf{D}^{*} & \mathbf{B}^{*} \otimes \mathbf{C}^{*} & \mathbf{A}^{*} \otimes \mathbf{D}^{*} & \mathbf{A}^{*} \otimes \mathbf{C}^{*}
\end{array}\right) \\
& \left(\begin{array}{cc}
\mathbf{G}^{T} & \mathbf{O} \\
\mathbf{O} & \mathbf{G}^{T}
\end{array}\right) \mathbf{K}_{2 M}\left(\begin{array}{cc}
\mathbf{G} & \mathbf{O} \\
\mathbf{O} & \mathbf{G}
\end{array}\right) \\
& =\left(\begin{array}{cccc}
\mathbf{K}_{M} & \mathbf{O} & \mathbf{O} & \mathbf{O} \\
\mathbf{O} & \mathbf{O} & \mathbf{K}_{M} & \mathbf{O} \\
\mathbf{O} & \mathbf{K} & \mathbf{O} & \mathbf{O} \\
\mathbf{O} & \mathbf{O} & \mathbf{O} & \mathbf{K}_{M}
\end{array}\right)
\end{aligned}
$$

the expressions of $\mathbf{C}_{\Pi_{1}}, \mathbf{C}_{\Pi_{2}}, \mathbf{C}_{\Pi_{2}, \Pi_{1}}, \mathbf{C}_{\Pi_{2}^{*}, \Pi_{1}}$, and $\mathbf{C}_{\Pi_{2}^{*}, \Pi_{2}}$ of Theorem 3 follow directly from (B.4).

\section{APPENDIX C \\ PROOF OF THEOREM 5}

We first prove that algorithms 2 and 3 satisfy the same firstorder perturbation expansion (5.7). For algorithm 2, we note that $\frac{\partial g_{2, T}(\theta)}{\partial \theta}=2 \operatorname{Tr}\left(\boldsymbol{\Pi}_{1, T} \mathbf{M}^{\prime}(\theta) \mathbf{\Pi}_{1, T} \mathbf{M}(\theta)\right)$ $-2 \Re\left[\operatorname{Tr}\left(\Pi_{2, T}^{*} \mathbf{M}^{\prime}(\theta) \mathbf{\Pi}_{2, T} \mathbf{M}^{*}(\theta)\right)\right]$

with $\mathbf{M}(\theta) \stackrel{\text { def }}{=} \mathbf{a}(\theta) \mathbf{a}^{H}(\theta)$ and $\mathbf{M}^{\prime}(\theta) \stackrel{\text { def }}{=}(d \mathbf{M}(\theta) / d \theta)$. Because $\theta_{k, T}$ satisfies $\left(\partial g_{2, T}(\theta) / \partial \theta\right)_{\mid \theta=\theta_{k, T}=\theta_{k}+\delta \theta_{k, T}}=0$, we straightforwardly obtain the following first-order perturbation expansion thanks to $\boldsymbol{\Pi}_{1, T}=\Pi_{1}+\delta \Pi_{1, T}, \Pi_{2, T}=\Pi_{2}+\delta \Pi_{2, T}$, $\mathbf{M}\left(\theta_{k, T}\right)=\mathbf{M}_{k}+\mathbf{M}_{k}^{\prime} \delta \theta_{k, T}+o\left(\delta \theta_{k, T}\right)$ and $\mathbf{M}^{\prime}\left(\theta_{k, T}\right)=$ $\mathbf{M}_{k}^{\prime}+\mathbf{M}_{k}^{\prime \prime} \delta \theta_{k, T}+o\left(\delta \theta_{k, T}\right)$ :

$$
\begin{aligned}
& {\left[\operatorname{Tr}\left(\boldsymbol{\Pi}_{1} \mathbf{M}^{\prime \prime}{ }_{k} \boldsymbol{\Pi}_{1} \mathbf{M}_{k}\right)-\Re\left(\operatorname{Tr}\left(\boldsymbol{\Pi}_{2}^{*} \mathbf{M}^{\prime \prime}{ }_{k} \boldsymbol{\Pi}_{2} \mathbf{M}_{k}^{*}\right)\right)\right.} \\
& \left.+\operatorname{Tr}\left(\boldsymbol{\Pi}_{1} \mathbf{M}_{k}^{\prime} \boldsymbol{\Pi}_{1} \mathbf{M}_{k}^{\prime}\right)-\Re\left(\operatorname{Tr}\left(\boldsymbol{\Pi}_{2}^{*} \mathbf{M}_{k}^{\prime} \boldsymbol{\Pi}_{2} \mathbf{M}_{k}^{*}\right)\right)\right] \delta \theta_{k, T} \\
& =\Re\left(\operatorname{Tr}\left(\delta \boldsymbol{\Pi}_{2, T}^{*} \mathbf{M}_{k}^{\prime} \boldsymbol{\Pi}_{2} \mathbf{M}_{k}^{*}\right)\right) \\
& +\Re\left(\operatorname{Tr}\left(\boldsymbol{\Pi}_{2}^{*} \mathbf{M}_{k}^{\prime} \delta \boldsymbol{\Pi}_{2, T} \mathbf{M}_{k}^{*}\right)\right) \\
& -\operatorname{Tr}\left(\delta \mathbf{\Pi}_{1, T} \mathbf{M}_{k}^{\prime} \boldsymbol{\Pi}_{1} \mathbf{M}_{k}\right) \\
& -\operatorname{Tr}\left(\boldsymbol{\Pi}_{1} \mathbf{M}_{k}^{\prime} \delta \boldsymbol{\Pi}_{1, T} \mathbf{M}_{k}\right)+o\left(\delta \boldsymbol{\Pi}_{1, T}\right)+o\left(\boldsymbol{\Pi}_{2, T}\right)
\end{aligned}
$$


with $\mathbf{M}_{k} \stackrel{\text { def }}{=} \mathbf{M}\left(\theta_{k}\right), \mathbf{M}_{k}^{\prime} \stackrel{\text { def }}{=} \mathbf{M}^{\prime}\left(\theta_{k}\right)$ and $\mathbf{M}_{k}^{\prime \prime} \stackrel{\text { def }}{=}$ $\left(d^{2} \mathbf{M}(\alpha) / d \alpha^{2}\right)_{\mid \alpha=\theta_{k}}$. Furthermore, we note that the sum of the first two terms of the left-hand side of (C.1) vanishes thanks to the identity $\tilde{\Pi} \tilde{\mathbf{a}}_{k}=\mathbf{0}$, which is equivalent to $\Pi_{1} \mathbf{a}_{k}+e^{-i \phi_{k}} \bar{\Pi}_{2} \mathbf{a}_{k}^{*}=\mathbf{0}$, and which implies

$$
\Pi_{1} \mathbf{M}_{k} \Pi_{1}=\Pi_{2} \mathbf{M}_{k}^{*} \Pi_{2}^{*} .
$$

Consequently

$$
\begin{aligned}
\operatorname{Tr} & \left(\boldsymbol{\Pi}_{1} \mathbf{M}^{{ }^{\prime}}{ }_{k} \Pi_{1} \mathbf{M}_{k}\right)-\Re\left(\operatorname{Tr}\left(\boldsymbol{\Pi}_{2}^{*} \mathbf{M}^{{ }^{\prime}}{ }_{k} \boldsymbol{\Pi}_{2} \mathbf{M}_{k}^{*}\right)\right) \\
& =\Re\left(\operatorname{Tr}\left(\left(\boldsymbol{\Pi}_{1} \mathbf{M}_{k} \boldsymbol{\Pi}_{1}-\boldsymbol{\Pi}_{2} \mathbf{M}_{k}^{*} \boldsymbol{\Pi}_{2}^{*}\right) \mathbf{M}^{\prime \prime}{ }_{k}\right)\right) \\
& =0 .
\end{aligned}
$$

Thus, (C.1) becomes (C.3), shown at the bottom of the page.

For algorithm 3, we note that

$$
\begin{aligned}
g_{3, T}(z)=\operatorname{Tr}\left(\boldsymbol{\Pi}_{1, T} \mathbf{M}(z) \boldsymbol{\Pi}_{1, T} \mathbf{M}(z)\right) & \\
& -\operatorname{Tr}\left(\boldsymbol{\Pi}_{2, T}^{*} \mathbf{M}(z) \boldsymbol{\Pi}_{2, T} \mathbf{M}\left(z^{-1}\right)\right)
\end{aligned}
$$

with $\mathbf{M}(z) \stackrel{\text { def }}{=} \mathbf{a}(z) \mathbf{a}^{T}\left(z^{-1}\right)$. By definition of algorithm 3 (see (4.7)), $z_{k, T}=\theta_{k}+\delta \theta_{k, T}$ is solution of

$$
\begin{aligned}
g_{3, T}\left(z_{k, T}\right) & =0 \text { with } \\
z_{k, T} & =r_{k, T} e^{i \theta_{k, T}}=z_{k}+\delta z_{k, T} \\
& =e^{i \theta_{k}}+\delta z_{k, T} .
\end{aligned}
$$

To relate $\delta \theta_{k, T}$ to $\delta \Pi_{1, T}, \delta \boldsymbol{\Pi}_{2, T}$, and $\delta \boldsymbol{\Pi}_{2, T}^{*}$, a second-order expansion of $\mathbf{a}(z), \boldsymbol{\Pi}_{1, T}$, and $\boldsymbol{\Pi}_{2, T}$ is required since the firstorder terms in $\delta \theta_{k, T}$ and $\delta r_{k, T}$ vanish, as noted in [13] for the standard root-MUSIC algorithm.

$$
\begin{aligned}
\mathbf{a}\left(z_{k, T}\right)= & \mathbf{a}_{k}+\mathbf{a}_{k}^{\prime} \delta \theta_{k, T}-i \mathbf{a}_{k}^{\prime} \delta r_{k, T} \\
& +\frac{1}{2} \mathbf{a}_{k}^{\prime \prime}\left(\delta \theta_{k, T}\right)^{2}-i \mathbf{a}_{k}^{\prime \prime} \delta \theta_{k, T} \delta r_{k, T} \\
& -\frac{1}{2} \mathbf{a}_{k}^{\prime \prime}\left(\delta r_{k, T}\right)^{2}+o_{2}\left(\delta \theta_{k, T}, \delta r_{k, T}\right) \\
\mathbf{a}\left(z_{k, T}^{-1}\right)= & \mathbf{a}_{k}^{*}+\mathbf{a}_{k}^{\prime *} \delta \theta_{k, T}-i \mathbf{a}_{k}^{\prime *} \delta r_{k, T} \\
& +\frac{1}{2} \mathbf{a}_{k}^{\prime \prime *}\left(\delta \theta_{k, T}\right)^{2}-i \mathbf{a}_{k}^{\prime \prime *} \delta \theta_{k, T} \delta r_{k, T} \\
& -\frac{1}{2} \mathbf{a}_{k}^{\prime \prime *}\left(\delta r_{k, T}\right)^{2}+o_{2}\left(\delta \theta_{k, T}, \delta r_{k, T}\right) \\
\Pi_{1, T}= & \Pi_{1}+\delta \boldsymbol{\Pi}_{1, T}+\delta^{2} \mathbf{\Pi}_{1, T}+o\left(\delta^{2} \mathbf{\Pi}_{1, T}\right) \\
\Pi_{2, T}= & \Pi_{2}+\delta \boldsymbol{\Pi}_{2, T}+\delta^{2} \mathbf{\Pi}_{2, T}+o\left(\delta^{2} \mathbf{\Pi}_{2, T}\right)
\end{aligned}
$$

with $\mathbf{a}_{k}^{\prime} \stackrel{\text { def }}{=}\left(d \mathbf{a}_{k} / d \theta_{k}\right)$ and $\mathbf{a}_{k}^{\prime \prime} \stackrel{\text { def }}{=}\left(d^{2} \mathbf{a}_{k} / d \theta_{k}^{2}\right)$, where $\left(\delta \boldsymbol{\Pi}_{i, T}\right)_{i=1,2}$ and $\left(\delta^{2} \boldsymbol{\Pi}_{i, T}\right)_{i=1,2}$ denote the first and second-order terms of the expansion of $\left(\Pi_{i, T}\right)_{i=1,2}$ with regard to $\delta \mathbf{R}_{\tilde{y}, T}$, and where $o_{2}\left(\delta \theta_{k, T}, \delta r_{k, T}\right)$ is a third-order term in $\left(\delta \theta_{k, T}, \delta r_{k, T}\right)$. Consequently

$$
\begin{aligned}
\mathbf{M}\left(z_{k, T}\right)= & \mathbf{M}_{k}+\mathbf{M}_{k}^{\prime} \delta \theta_{k, T}-i \mathbf{M}_{k}^{\prime} \delta r_{k, T} \\
& +\frac{1}{2} \mathbf{M}_{k}^{\prime \prime}\left(\delta \theta_{k, T}\right)^{2}-i \mathbf{M}_{k}^{\prime \prime} \delta \theta_{k, T} \delta r_{k, T} \\
& \left.-\frac{1}{2} \mathbf{M}_{k}^{\prime \prime}\left(\delta r_{k, T}\right)^{2}+o_{2}\left(\delta \theta_{k, T}, \delta r_{k, T}\right)\right) \\
\mathbf{M}\left(z_{k, T}^{-1}\right)= & \mathbf{M}_{k}^{*}+\mathbf{M}_{k}^{\prime *} \delta \theta_{k, T}-i \mathbf{M}_{k}^{\prime *} \delta r_{k, T} \\
& +\frac{1}{2} \mathbf{M}_{k}^{\prime \prime *}\left(\delta \theta_{k, T}\right)^{2}-i \mathbf{M}_{k}^{\prime \prime *} \delta \theta_{k, T} \delta r_{k, T} \\
& \left.-\frac{1}{2} \mathbf{M}_{k}^{\prime \prime *}\left(\delta r_{k, T}\right)^{2}+o_{2}\left(\delta \theta_{k, T}, \delta r_{k, T}\right)\right) .
\end{aligned}
$$

Inserting these second-order expansions of $\boldsymbol{\Pi}_{1, T}, \boldsymbol{\Pi}_{2, T}$, $\mathbf{M}\left(z_{k, T}\right)$, and $\mathbf{M}\left(z_{k, T}^{-1}\right)$ in the expression (C.4) of $g_{2, T}\left(z_{k, T}\right)$ and using identity (C.2) and identity

$$
\mathbf{a}_{k}^{H} \delta \Pi_{1, T} \mathbf{a}_{k}+\Re\left(\mathbf{a}_{k}^{H} \delta \boldsymbol{\Pi}_{2, T} \mathbf{a}_{k}^{*} e^{-i \phi_{k}}\right)=0
$$

deduced from identity $\tilde{\mathbf{a}}_{k}^{H} \delta \tilde{\mathbf{\Pi}}_{T} \tilde{\mathbf{a}}_{k}=0$ (issued from $\tilde{\Pi} \tilde{\mathbf{a}}_{k}=\mathbf{0}$ and $\delta \tilde{\boldsymbol{\Pi}}_{T} \tilde{\mathbf{a}}_{k}+\tilde{\boldsymbol{\Pi}} \delta \tilde{\mathbf{a}}_{k, T}=\mathbf{0}$ ), one can check that the first-order terms in $\delta \theta_{k, T}$ and $\delta r_{k, T}$ vanish, and the following expression of $g_{3, T}\left(z_{k, T}\right)$ of (C.4) is obtained after simple, but tedious, algebra manipulations:

$$
\begin{aligned}
& g_{3, T}\left(z_{k, T}\right)=\frac{1}{2}\left\{\left(\operatorname{Tr}\left(\boldsymbol{\Pi}_{1} \mathbf{M}_{k}^{\prime} \boldsymbol{\Pi}_{1} \mathbf{M}_{k}^{\prime}\right)\right.\right. \\
& \left.-\Re\left(\operatorname{Tr}\left(\boldsymbol{\Pi}_{2}^{*} \mathbf{M}_{k}^{\prime} \boldsymbol{\Pi}_{2} \mathbf{M}_{k}^{\prime *}\right)\right)\right)\left(\left(\delta \theta_{k, T}\right)^{2}-\left(\delta r_{k, T}\right)^{2}\right) \\
& +2\left(\operatorname{Tr}\left(\delta \boldsymbol{\Pi}_{1, T} \mathbf{M}_{k} \boldsymbol{\Pi}_{1} \mathbf{M}_{k}^{\prime}\right)\right. \\
& -\Re\left(\operatorname{Tr}\left(\delta \mathbf{\Pi}_{2, T}^{*} \mathbf{M}_{k} \boldsymbol{\Pi}_{2} \mathbf{M}_{k}^{\prime *}\right)\right) \\
& +\operatorname{Tr}\left(\boldsymbol{\Pi}_{1} \mathbf{M}_{k} \delta \boldsymbol{\Pi}_{1, T} \mathbf{M}_{k}^{\prime}\right) \\
& \left.-\Re\left(\operatorname{Tr}\left(\boldsymbol{\Pi}_{2}^{*} \mathbf{M}_{k} \delta \boldsymbol{\Pi}_{2, T} \mathbf{M}_{k}^{\prime *}\right)\right)\right) \delta \theta_{k, T} \\
& +2\left(\operatorname{Tr}\left(\boldsymbol{\Pi}_{1} \mathbf{M}_{k} \delta^{2} \boldsymbol{\Pi}_{1, T} \mathbf{M}_{k}\right)\right. \\
& \left.-\Re\left(\operatorname{Tr}\left(\boldsymbol{\Pi}_{2}^{*} \mathbf{M}_{k} \delta^{2} \boldsymbol{\Pi}_{2, T} \mathbf{M}_{k}^{*}\right)\right)\right\} \\
& -2 i\left\{\operatorname{Tr}\left(\delta \boldsymbol{\Pi}_{1, T} \mathbf{M}_{k}^{\prime} \boldsymbol{\Pi}_{1} \mathbf{M}_{k}\right)\right. \\
& +\operatorname{Tr}\left(\boldsymbol{\Pi}_{1} \mathbf{M}_{k}^{\prime} \delta \boldsymbol{\Pi}_{1, T} \mathbf{M}_{k}\right) \\
& -\Re\left(\operatorname{Tr}\left(\delta \mathbf{\Pi}_{2, T}^{*} \mathbf{M}_{k}^{\prime} \mathbf{\Pi}_{2} \mathbf{M}_{k}^{*}\right)\right. \\
& -\Re\left(\operatorname{Tr}\left(\mathbf{\Pi}_{2}^{*} \mathbf{M}_{k}^{\prime} \delta \mathbf{\Pi}_{2, T} \mathbf{M}_{k}^{*}\right)\right. \\
& +\left(\operatorname{Tr}\left(\boldsymbol{\Pi}_{1} \mathbf{M}_{k}^{\prime} \boldsymbol{\Pi}_{1} \mathbf{M}_{k}^{\prime}\right)\right. \\
& \left.\left.-\Re\left(\operatorname{Tr}\left(\boldsymbol{\Pi}_{2}^{*} \mathbf{M}_{k}^{\prime} \boldsymbol{\Pi}_{2} \mathbf{M}_{k}^{\prime *}\right)\right)\right) \delta \theta_{k, T}\right\} \delta r_{k, T} \\
& +o_{2}\left(\delta \theta_{k, T}, \delta r_{k, T}\right) \text {. }
\end{aligned}
$$

Since the different matrices $\mathbf{M}$ are composed of sums of rank-one matrices and that matrices $\boldsymbol{\Pi}_{1}, \delta \boldsymbol{\Pi}_{1, T}$ and $\delta^{2} \boldsymbol{\Pi}_{1, T}$, (respectively, $\Pi_{2}, \delta \Pi_{2, T}$, and $\delta^{2} \Pi_{2, T}$ ) are Hermitian (respectively, complex symmetric) structured, one can check that the

$$
\begin{aligned}
\delta \theta_{k, T}= & \frac{\Re\left(\operatorname{Tr}\left(\delta \boldsymbol{\Pi}_{2, T}^{*} \mathbf{M}_{k}^{\prime} \boldsymbol{\Pi}_{2} \mathbf{M}_{k}^{*}+\boldsymbol{\Pi}_{2}^{*} \mathbf{M}_{k}^{\prime} \delta \boldsymbol{\Pi}_{2, T} \mathbf{M}_{k}^{*}\right)\right)-\operatorname{Tr}\left(\delta \boldsymbol{\Pi}_{1, T} \mathbf{M}_{k}^{\prime} \boldsymbol{\Pi}_{1} \mathbf{M}_{k}+\boldsymbol{\Pi}_{1} \mathbf{M}_{k}^{\prime} \delta \boldsymbol{\Pi}_{1, T} \mathbf{M}_{k}\right)}{\operatorname{Tr}\left(\boldsymbol{\Pi}_{1} \mathbf{M}_{k}^{\prime} \boldsymbol{\Pi}_{1} \mathbf{M}_{k}^{\prime}\right)-\Re\left(\operatorname{Tr}\left(\boldsymbol{\Pi}_{2}^{*} \mathbf{M}_{k}^{\prime} \boldsymbol{\Pi}_{2} \mathbf{M}_{k}^{\prime *}\right)\right)} \\
& +o\left(\delta \boldsymbol{\Pi}_{1, T}\right)+o\left(\delta \boldsymbol{\Pi}_{2, T}\right) .
\end{aligned}
$$


first four lines within the braces in (C.6) are real, while the last two lines in the second brace are purely imaginary. By setting the imaginary part of this expansion equal to zero $\left(z_{k, T}\right.$ is a root of $\left.g_{3, T}(z)\right)$, (C.3) is found.

Remark: With different cost functions, we note the similarity of behavior of our algorithms 1 and 2, with the standard MUSIC and root-MUSIC algorithms analyzed in [13]: In the two cases, the asymptotic distributions of the DOA estimates given by the MUSIC and the associated root-MUSIC algorithm are identical. Furthermore the second-order terms in $\delta^{2} \boldsymbol{\Pi}_{1, T}$ and $\delta^{2} \boldsymbol{\Pi}_{2, T}$ are not used for the derivation of $\delta \theta_{k, T}$ (they would be used in the derivation of $\delta r_{k, T}$, which is not studied in this paper) for the two root-MUSIC algorithms.

Considering algorithm 1 , the estimates $\theta_{k, T}$ and $\phi_{k, T}$ are solutions of the global minimization

$$
\left(\theta_{k, T}, \phi_{k, T}\right)=\arg \min _{\theta, \phi} \tilde{g}_{1, T}(\theta, \phi)
$$

with $\tilde{g}_{1, T}(\theta, \phi)=\tilde{\mathbf{a}}^{H}(\theta, \phi) \tilde{\mathbf{\Pi}}_{T} \tilde{\mathbf{a}}(\theta, \phi)=\operatorname{Tr}\left(\tilde{\mathbf{\Pi}}_{T} \mathbf{M}_{\theta, \phi}\right)$, where $\mathbf{M}_{\theta, \phi} \stackrel{\text { def }}{=} \tilde{\mathbf{a}}(\theta, \phi) \tilde{\mathbf{a}}(\theta, \phi)^{H}$. Because $\left(\theta_{k, T}, \phi_{k, T}\right)$ satisfies

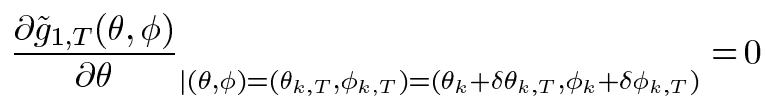

and

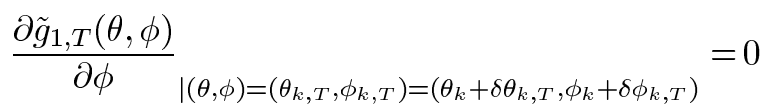

we straightforwardly obtain the following first-order perturbation expansion

$$
\begin{aligned}
\operatorname{Tr}\left(\tilde{\mathbf{\Pi}} \mathbf{M}_{\theta, \theta}^{\prime \prime}\right) \delta \theta_{k, T}+\operatorname{Tr}\left(\tilde{\mathbf{\Pi}} \mathbf{M}_{\theta, \phi}^{\prime \prime}\right) \delta \phi_{k, T}+\operatorname{Tr}\left(\delta \tilde{\mathbf{\Pi}}_{T} \mathbf{M}_{\theta}^{\prime}\right) & =0 \\
\operatorname{Tr}\left(\tilde{\mathbf{\Pi}} \mathbf{M}_{\theta, \phi}^{\prime \prime}\right) \delta \theta_{k, T}+\operatorname{Tr}\left(\tilde{\mathbf{\Pi}} \mathbf{M}_{\phi, \phi}^{\prime \prime}\right) \delta \phi_{k, T}+\operatorname{Tr}\left(\delta \tilde{\mathbf{\Pi}}_{T} \mathbf{M}_{\phi}^{\prime}\right) & =0
\end{aligned}
$$

with $\mathbf{M}_{\theta}^{\prime} \stackrel{\text { def }}{=}\left(\partial \mathbf{M}_{\theta, \phi} / \partial \theta\right), \mathbf{M}_{\phi}^{\prime} \stackrel{\text { def }}{=}\left(\partial \mathbf{M}_{\theta, \phi} / \partial \phi\right)$, $\mathbf{M}_{\theta, \theta}^{\prime \prime} \stackrel{\text { def }}{=}\left(\partial^{2} \mathbf{M}_{\theta, \phi} / \partial \theta^{2}\right), \mathbf{M}_{\theta, \phi}^{\prime \prime} \stackrel{\text { def }}{=}\left(\partial^{2} \mathbf{M}_{\theta, \phi} / \partial \theta \partial \phi\right)$, and $\mathbf{M}_{\phi, \phi}^{\prime \prime} \stackrel{\text { def }}{=}\left(\partial^{2} \mathbf{M}_{\theta, \phi} / \partial \phi^{2}\right)$ associated with the source $k$. Noting that $\operatorname{Tr}\left(\tilde{\mathbf{\Pi}} \mathbf{M}_{i, j}^{\prime \prime}\right)=2 \Re\left(\tilde{\mathbf{a}}_{i, k}^{H} \tilde{\boldsymbol{\Pi}} \tilde{\mathbf{a}}_{j, k}^{\prime}\right), i, j=\theta, \phi$ which is denoted by $\alpha_{i, j}^{(k)}$, (C.7) gives

$$
\begin{aligned}
\delta \theta_{k, T}= & \frac{-1}{\alpha_{\theta, \theta}^{(k)} \alpha_{\phi, \phi}^{(k)}-\left(\alpha_{\theta, \phi}^{(k)}\right)^{2}} \\
& \times\left(\alpha_{\phi, \phi}^{(k)} \operatorname{Tr}\left(\delta \tilde{\boldsymbol{\Pi}}_{T} \mathbf{M}_{\theta}^{\prime}\right)-\alpha_{\theta, \phi}^{(k)} \operatorname{Tr}\left(\delta \tilde{\boldsymbol{\Pi}}_{T} \mathbf{M}_{\phi}^{\prime}\right)\right)+o\left(\delta \tilde{\boldsymbol{\Pi}}_{T}\right)
\end{aligned}
$$

where $\operatorname{Tr}\left(\delta \tilde{\mathbf{\Pi}}_{T} \mathbf{M}_{i}^{\prime}\right)=\left(\tilde{\mathbf{a}}^{\prime_{i, k}^{T}}{ }^{T} \otimes \tilde{\mathbf{a}}_{k}^{H}+\tilde{\mathbf{a}}_{k}^{T} \otimes \tilde{\mathbf{a}}^{\prime}{ }_{i, k}^{H}\right) \operatorname{vec}\left(\delta \tilde{\mathbf{\Pi}}_{T}\right)$, $i=\theta, \phi$. Consequently, the Jacobian matrix $\mathbf{D}_{\Theta}^{\operatorname{Alg}_{1}}$ of the mapping that associates $\Theta_{T}$ to $\tilde{\Pi}_{T}$ is given by $\mathbf{D}_{\Theta}^{\mathrm{Alg}_{1}}=\left(\mathbf{d}_{1}, \ldots, \mathbf{d}_{K}\right)^{T}$ with

$$
\begin{aligned}
\mathbf{d}_{k}^{T}= & \frac{-1}{\alpha_{\theta, \theta}^{(k)} \alpha_{\phi, \phi}^{(k)}-\left(\alpha_{\theta, \phi}^{(k)}\right)^{2}} \\
& \times\left(\alpha_{\phi, \phi}^{(k)}\left(\tilde{\mathbf{a}}_{\theta, k}^{T} \otimes \tilde{\mathbf{a}}_{k}^{H}+\tilde{\mathbf{a}}_{k}^{T} \otimes \tilde{\mathbf{a}}_{\theta, k}^{H}\right)\right. \\
& \left.\quad-\alpha_{\theta, \phi}^{(k)}\left(\tilde{\mathbf{a}}_{\phi, k}^{T} \otimes \tilde{\mathbf{a}}_{k}^{H}+\tilde{\mathbf{a}}_{k}^{T} \otimes \tilde{\mathbf{a}}_{\phi, k}^{H}\right)\right) .
\end{aligned}
$$

Because $\tilde{\boldsymbol{\Pi}}_{T}$ is asymptotically Gaussian distributed with first covariance $\mathbf{C}_{\tilde{\Pi}}, \Theta_{T}$ is also asymptotically Gaussian distributed thanks to the standard theorem of continuity (see, e.g.,
[18, p. 122]) with covariance $\mathbf{C}_{\Theta}=\mathbf{D}_{\Theta}^{\mathrm{Alg}_{1}} \mathbf{C}_{\tilde{\Pi}}\left(\mathbf{D}_{\Theta}^{\mathrm{Alg}_{1}}\right)^{H}$. Using, the expression (5.1) of $\mathbf{C}_{\tilde{\Pi}}$, the expressions (5.4) and (5.5) of $\mathbf{C}_{\Theta}$ are straightforwardly deduced after simple but tedious algebra manipulations thanks to the identities $(\mathbf{a} \otimes \mathbf{b}) \mathbf{K}_{2 M}=(\mathbf{b} \otimes \mathbf{a})$ for all $2 M \times 1$ vectors $\mathbf{a}, \mathbf{b}$, $\tilde{\mathbf{\Pi}} \mathbf{a}=\mathbf{0}$ and $\tilde{\mathbf{a}}^{H} \mathbf{J} \tilde{\Pi}^{*}=\mathbf{0}^{T}$. In the case of a single source, $\tilde{\mathbf{U}}=\left(1 / 2\|\mathbf{a}\|^{2}\right)\left(\left(\sigma_{n}^{2} / \sigma_{1}^{2}\right)+\left(1 / 2\|\mathbf{a}\|^{2}\right)\left(\sigma_{n}^{4} / \sigma_{1}^{4}\right)\right)(\tilde{\mathbf{a}} / \sqrt{2}\|\mathbf{a}\|)$ $(\tilde{\mathbf{a}} / \sqrt{2}\|\mathbf{a}\|)^{H}$ and (5.6) is straightforwardly deduced as well.

\section{APPENDIX D}

PROOF OF THEOREM 7

Because $\theta_{k, T}$ satisfies $\left(\partial g_{5, T}(\theta) / \partial \theta\right)_{\mid \theta=\theta_{k, T}=\theta_{k}+\delta \theta_{k, T}}=0$, we straightforwardly obtain the following first-order perturbation expansion thanks to $\tilde{\boldsymbol{\Pi}}_{T}=\tilde{\boldsymbol{\Pi}}+\delta \tilde{\boldsymbol{\Pi}}_{T}$, where we have used the identity $\operatorname{Tr}(\mathbf{A B C D})=\operatorname{vec}^{T}\left(\mathbf{A}^{T}\right)\left(\mathbf{D}^{T} \otimes \mathbf{B}\right) \operatorname{vec}(\mathbf{C})[19$, th. 7.17]

$$
\begin{aligned}
& \delta \theta_{k, T}=\frac{\operatorname{vec}^{H}(\mathbf{W})\left(\overline{\mathbf{A}}_{k}^{T} \otimes \overline{\mathbf{A}}_{k}^{\prime H}+\overline{\mathbf{A}}_{k}^{T} \otimes \overline{\mathbf{A}}_{k}^{H}\right) \operatorname{vec}\left(\delta \tilde{\mathbf{\Pi}}_{T}\right)}{2 \operatorname{Tr}\left(\mathbf{W} \overline{\mathbf{A}}_{k}^{\prime H} \tilde{\Pi}_{\mathbf{A}_{k}^{\prime}}^{\prime}\right)} \\
& +o\left(\delta \tilde{\boldsymbol{\Pi}}_{T}\right) .
\end{aligned}
$$

And because $\tilde{\Pi}_{T}$ is asymptotically Gaussian distributed, $\Theta_{T}$ is also asymptotically Gaussian distributed thanks to the standard theorem of continuity (see e.g., [18, p. 122]) with covariance:

$$
\left(\mathbf{C}_{\Theta}\right)_{k, l}=\frac{1}{\beta_{k} \beta_{l}} \operatorname{vec}^{H}(\mathbf{W}) \mathbf{M}_{k} \mathbf{C}_{\tilde{\Pi}} \mathbf{M}_{l}^{H} \operatorname{vec}(\mathbf{W})
$$

with $\beta_{k} \stackrel{\text { def }}{=} 2 \operatorname{Tr}\left(\mathbf{W} \overline{\mathbf{A}}_{k}^{\prime H} \tilde{\Pi} \overline{\mathbf{A}}_{k}^{\prime}\right)$ and $\mathbf{M}_{k} \stackrel{\text { def }}{=} \overline{\mathbf{A}}_{k}^{T} \otimes$ $\overline{\mathbf{A}}_{k}^{\prime H}+\overline{\mathbf{A}}^{\prime}{ }_{k}^{T} \otimes \overline{\mathbf{A}}_{k}^{H}$. Using the alternative expression $(1 / 2) \mathbf{L}_{M}\left(\tilde{\mathbf{\Pi}}^{*} \otimes \tilde{\mathbf{U}}+\tilde{\mathbf{U}}^{*} \otimes \tilde{\mathbf{\Pi}}\right) \mathbf{L}_{M}$ of $\mathbf{C}_{\tilde{\Pi}}$ given by (5.1) where $\mathbf{L}_{M} \stackrel{\text { def }}{=}\left(\mathbf{I}_{4 M^{2}}+\mathbf{K}_{2 M}\left(\mathbf{J}_{M} \otimes \mathbf{J}_{M}\right)\right)$, we obtain thanks to straightforward algebra manipulations

$$
\begin{aligned}
\mathbf{M}_{k} \mathbf{C}_{\tilde{\Pi}} \mathbf{M}_{l}^{H}=\frac{1}{2} \mathbf{L}_{4}( & \left(\overline{\mathbf{A}}_{k}^{T} \tilde{\mathbf{U}}^{*} \overline{\mathbf{A}}_{l}^{*}\right) \otimes\left(\overline{\mathbf{A}}_{k}^{\prime H} \tilde{\mathbf{\Pi}} \overline{\mathbf{A}}_{l}^{\prime}\right) \\
& \left.+\left(\overline{\mathbf{A}}_{k}{ }_{k}^{T} \tilde{\boldsymbol{\Pi}}^{*} \overline{\mathbf{A}}^{*}{ }_{l}^{\prime}\right) \otimes\left(\overline{\mathbf{A}}_{k}^{H} \tilde{\mathbf{U}} \overline{\mathbf{A}}_{l}\right)\right) \mathbf{L}_{4}
\end{aligned}
$$

and because

$$
\mathbf{L}_{4} \operatorname{vec}(\mathbf{W})=\left(\begin{array}{c}
w_{1,1}+w_{2,2} \\
2 w_{1,2}^{*} \\
2 w_{1,2} \\
w_{1,1}+w_{2,2}
\end{array}\right)
$$

and $\beta=2\left(w_{1,1}+w_{2,2}\right) \mathbf{a}_{k}^{\prime H} \mathbf{\Pi}_{1} \mathbf{a}_{k}^{\prime}$, expression (5.10) is proved.

Expressing the matrix

$\mathbf{C} \stackrel{\text { def }}{=}\left(\overline{\mathbf{A}}_{k}^{T} \tilde{\mathbf{U}}^{*} \overline{\mathbf{A}}_{l}^{*}\right) \otimes\left(\overline{\mathbf{A}}_{k}^{\prime H} \tilde{\mathbf{\Pi}} \overline{\mathbf{A}}_{l}^{\prime}\right)+\left(\overline{\mathbf{A}}_{k}^{\prime T} \tilde{\boldsymbol{\Pi}}^{*} \overline{\mathbf{A}}_{l}^{\prime *}\right) \otimes\left(\overline{\mathbf{A}}_{k}^{H} \tilde{\mathbf{U}} \overline{\mathbf{A}}_{l}\right)$ of (5.10) as a function of $\mathbf{a}_{k}, \Pi_{1}$ and $\boldsymbol{\Pi}_{2}$, we obtain after simple but tedious algebra manipulations

$$
\mathbf{C}=\left(\begin{array}{cccc}
2 \alpha & \beta & \beta^{*} & 0 \\
\beta^{*} & 2 \alpha & 0 & \beta^{*} \\
\beta & 0 & 2 \alpha & \beta \\
0 & \beta & \beta^{*} & 2 \alpha
\end{array}\right)
$$

with $\alpha \stackrel{\text { def }}{=}\left(\mathbf{a}_{k}^{H} \mathbf{U}_{1} \mathbf{a}_{k}\right)\left(\mathbf{a}^{\prime}{ }_{k}^{H} \mathbf{\Pi}_{1} \mathbf{a}_{k}^{\prime}\right)$ and $\beta \stackrel{\text { def }}{=}$ $\left(\mathbf{a}_{k}^{H} \mathbf{U}_{2} \mathbf{a}_{k}^{*}\right)\left(\mathbf{a}_{k}^{\prime H} \mathbf{\Pi}_{1} \mathbf{a}_{k}^{\prime}\right)$. Consequently, (5.10) becomes

$$
\left(\mathbf{C}_{\Theta}\right)_{k, k}=\frac{1}{2 \alpha_{k}^{2}} 4\left[\alpha\left(1+|z|^{2}\right)+2 \Re(\beta z)\right]
$$


which is minimum for $z_{\mathrm{opt}}=-\left(\beta^{*} / \alpha\right)=$ $-\left(\mathbf{a}_{k}^{T} \mathbf{U}_{2}^{*} \mathbf{a}_{k} / \mathbf{a}_{k}^{H} \mathbf{U}_{1} \mathbf{a}_{k}\right)$.

The associated minimum value of $\left(\mathbf{C}_{\Theta}\right)_{k, k}$ is

$$
\begin{aligned}
\min _{z}\left(\mathbf{C}_{\Theta}\right)_{k, k} & =\frac{1}{2 \alpha_{k}^{2}} \frac{4}{\alpha}\left(\alpha^{2}-|\beta|^{2}\right) \\
& =\frac{\left(\mathbf{a}_{k}^{H} \mathbf{U}_{1} \mathbf{a}_{k}\right)^{2}-\left|\mathbf{a}_{k}^{H} \mathbf{U}_{2} \mathbf{a}_{k}^{*}\right|^{2}}{2\left(\mathbf{a}_{k}^{\prime H} \mathbf{\Pi}_{1} \mathbf{a}_{k}^{\prime}\right)\left(\mathbf{a}_{k}^{H} \mathbf{U}_{1} \mathbf{a}_{k}\right)}
\end{aligned}
$$

and the proof is completed.

We note that replacement of $\mathbf{W}$ by an arbitrary consistent estimate that satisfies $\mathbf{W}_{T}=\mathbf{W}+O\left(\mathbf{R}_{\tilde{y}}-\mathbf{R}_{\tilde{y}, T}\right)$ has no effect on the asymptotic variance of the weighted MUSIC estimates because the first order perturbation (D.1) is preserved.

\section{APPENDIX E \\ PROOF OF COROLLARY 1}

For the single source case, we obtain from the expressions of $\tilde{\mathbf{U}}$ given at the end of Appendix D

$$
\begin{aligned}
\mathbf{U}_{1}= & \frac{\sigma_{n}^{2}}{\sigma_{1}^{2}\left\|\mathbf{a}_{1}\right\|^{4}\left(1-\rho_{1}^{2}\right)}\left(1+\frac{\sigma_{n}^{2}}{\sigma_{1}^{2}\left\|\mathbf{a}_{1}\right\|^{2}} \frac{1+\rho_{1}^{2}}{1-\rho_{1}^{2}}\right) \mathbf{a}_{1} \mathbf{a}_{1}^{H} \\
\mathbf{U}_{2}= & -\frac{\rho_{1} \sigma_{n}^{2}}{\sigma_{1}^{2}\left\|\mathbf{a}_{1}\right\|^{4}\left(1-\rho_{1}^{2}\right)} \\
& \times\left(1+\frac{\sigma_{n}^{2}}{\sigma_{1}^{2}\left\|\mathbf{a}_{1}\right\|^{2}} \frac{2}{1-\rho_{1}^{2}}\right) e^{i \phi_{1}} \mathbf{a}_{1} \mathbf{a}_{1}^{T} .
\end{aligned}
$$

Then, using these values in expression (D.2) of $\min _{z}\left(\mathbf{C}_{\Theta}\right)_{k, k}$ we obtain after tedious but simple algebra manipulations

$$
\begin{aligned}
\min _{z} C_{\theta_{1}} & =\frac{1}{\alpha} \frac{\left(\mathbf{a}_{1}^{H} \mathbf{U}_{1} \mathbf{a}_{1}\right)^{2}-\left|\mathbf{a}_{1}^{H} \mathbf{U}_{2} \mathbf{a}_{1}^{*}\right|^{2}}{\left(\mathbf{a}_{1}^{H} \mathbf{U}_{1} \mathbf{a}_{1}\right)} \\
& =\frac{1}{\alpha}\left[\frac{2 r_{1}^{-1}+\left\|\mathbf{a}_{1}\right\|^{-2} r_{1}^{-2}+\left\|\mathbf{a}_{1}\right\|^{2}-\left\|\mathbf{a}_{1}\right\|^{2} \rho_{1}^{2}}{\left\|\mathbf{a}_{1}\right\|^{2} r_{1}+1+\left(1-\left\|\mathbf{a}_{1}\right\|^{2} r_{1}\right) \rho_{1}^{2}}\right]
\end{aligned}
$$

where $r_{1} \stackrel{\text { def }}{=}\left(\sigma_{1}^{2} / \sigma_{n}^{2}\right)$, which is the expression of the noncircular Gaussian Cramer-Rao bound proved in [4].

\section{REFERENCES}

[1] P. Stoica and A. Nehorai, "MUSIC, maximum likelihood, and Cramer-Rao bound," IEEE Trans. Acoust. Speech, Signal Process., vol. 37, no. 5, pp. 720-741, May 1989.

[2] - "MUSIC, maximum likelihood, and Cramer-Rao bound: Further results and comparisons," IEEE Trans. Acoust. Speech, Signal Process., vol. 38, no. 12, pp. 2140-2150, Dec. 1990.

[3] J. P. Delmas, "Asymptotically minimum variance second-order estimation for noncircular signals with application to DOA estimation," IEEE Trans. Signal Process., vol. 52, no. 5, pp. 1235-1241, May 2004.

[4] J. P. Delmas and H. Abeida, "Stochastic Cramer-Rao bound for noncircular signals with application to DOA estimation," IEEE Trans. Signal Process., vol. 52, no. 11, pp. 3192-3199, Nov. 2004.

[5] P. Gounon, C. Adnet, and J. Galy, "Localization angulaire de signaux non circulaires," Traitement du Signal, vol. 15, no. 1, pp. 17-23, 1998.

[6] P. Chargé, Y. Wang, and J. Saillard, "A noncircular sources direction finding method using polynomial rooting," Signal Process., vol. 81, pp. 1765-1770, 2001.
[7] M. Haardt and F. Römer, "Enhancements of unitary esprit for noncircular sources," in Proc. Iint. Conf. Acoustics, Speech, Signal Processing (ICASSP), vol. 2, Montreal, QC, Canada, May 2004, pp. 101-104.

[8] A. Zoubir, P. Chargé, and Y. Wang, "Non circular sources localization with ESPRIT," presented at the 6th Eur. Conf. Wireless Technology, Munich, Germany, Oct. 2003.

[9] D. B. Williams and D. H. Johnson, "On resolving $2 M-1$ narrowband signals with an $M$ sensor uniform linear array," IEEE Trans. Signal Process., vol. 40, no. 3, pp. 707-711, Mar. 1992.

[10] P. Stoica and A. Eriksson, "MUSIC estimation of real-valued sine-wave frequencies," Signal Process., vol. 42, pp. 139-146, 1995.

[11] P. Stoica, A. Eriksson, and T. Söderström, "Optimally weighted MUSIC for frequency estimation," SIAM J. Matrix Anal. Appl., vol. 16, no. 3, pp. 811-827, Jul. 1995.

[12] J. P. Delmas, "Asymptotic performance of second-order algorithms," IEEE Trans. Signal Process., vol. 50, no. 1, pp. 49-57, Jan. 2002.

[13] H. Krim, P. Forster, and G. Proakis, "Operator approach to performance analysis of root-MUSIC and root-min-norm," IEEE Trans. Signal Process., vol. 40, no. 7, pp. 1687-1696, Jul. 1992.

[14] B. Porat and B. Friedlander, "Performance analysis of parameter estimation algorithms based on high-order moments," Int. J. Adapt. Control Signal Process., vol. 3, pp. 191-229, 1989.

[15] J. F. Cardoso and E. Moulines, "Invariance of subspace based estimator," IEEE Trans. Signal Process., vol. 48, no. 9, pp. 2495-2505, Sep. 2000.

[16] B. Friedlander and B. Porat, "Asymptotically optimal estimation of MA and ARMA parameters of non-Gaussian processes from high-order moments," IEEE Trans. Autom. Control, vol. 35, no. 1, pp. 27-35, Jan. 1990.

[17] T. Kato, Perturbation Theory for Linear Operators. Berlin, Germany: Springer-Verlag, 1995.

[18] R. J. Serfling, Approximation Theorems of Mathematical Statistics. New York: Wiley, 1980.

[19] J. R. Schott, Matrix Analysis for Statistics. New York: Wiley, 1980.

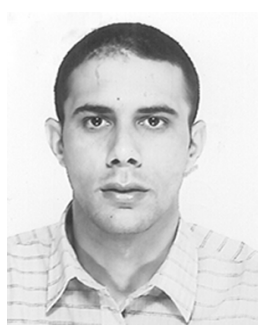

Habti Abeida was born in Settat, Morocco, on October 20, 1977. He received the Mastery Engineering degrees in applied mathematics from Hassan II University, Casablanca, Morocco, and René Descartes University, Paris, France, in 2000 and 2001, respectively, and the Master's degree in statistics from Pierre et Marie Curie University, Paris, France, in 2002. He is currently working towards the Ph.D. degree in applied mathematics and digital communications in the Institut National des Télecommunications, Evry, France.

His research interests are in statistical signal processing.

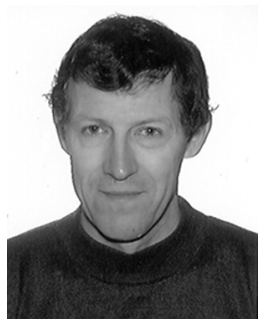

Jean-Pierre Delmas (M'00-SM'06) was born in France in 1950. He received the Engineering degree from Ecole Centrale de Lyon, Lyon, France, in 1973, the Certificat d'études supérieures from the Ecole Nationale Supérieure des Télécommunications, Paris, France, in 1982, and the Habilitation à Diriger des Recherches (HDR) degree from the University of Paris, Orsay, France, in 2001.

Since 1980, he has been with the Institut National des Télécommunications, Evry, France, where he is presently a Professor in the CITI Department and Assistant Director of UMR-CNRS 5157 (Samovar) laboratory. His teaching and research interests are in the areas of statistical signal processing with application to communications and antenna array.

Dr. Delmas is currently an Associate Editor for the IEEE TRANSACTIONS ON SIGNAL PROCESSING. 